\title{
VERIFL: Communication-efficient and Fast Verifiable Aggregation for Federated Learning
}

\author{
Xiaojie Guo, Zheli Liu*, Jin Li, Jiqiang Gao, Boyu Hou, Changyu Dong, and Thar Baker
}

\begin{abstract}
Federated learning (FL) enables a large number of clients to collaboratively train a global model through sharing their gradients in each synchronized epoch of local training. However, a centralized server used to aggregate these gradients can be compromised and forge the result in order to violate privacy or launch other attacks, which incurs the need to verify the integrity of aggregation. In this work, we explore how to design communication-efficient and fast verifiable aggregation in FL. We propose VERIFL, a verifiable aggregation protocol, with $O(N)$ (dimension-independent) communication and $O(N+d)$ computation for verification in each epoch, where $N$ is the number of clients and $d$ is the dimension of gradient vectors. Since $d$ can be large in some real-world FL applications (e.g., $100 \mathrm{~K}$ ), our dimension-independent communication is especially desirable for clients with limited bandwidth and high-dimensional gradients. In addition, the proposed protocol can be used in the FL setting where secure aggregation is needed or there is a subset of clients dropping out of protocol execution. Experimental results indicate that our protocol is efficient in these settings.
\end{abstract}

Index Terms-federated learning, verifiable aggregation, linearly homomorphic hash, commitment, machine learning.

\section{INTRODUCTION}

Federated learning (FL) [1], [2], [3] has become one of the most popular paradigms for distributed machine learning nowadays. It facilitates the usage of sensitive datasets distributed among a large number of clients, which may be mobile phones, other mobile devices, or sensors, without collecting their data. In FL, to train a global model, a subset of users are instructed to upload their local parameters in each synchronous epoch (Figure 1(a)). To update the global model, a centralized server is adopted to aggregate the parameters received from these users and sends back the updated global model to them. All users will update their local model according to the global one and such training process will continue until model convergence.

Despite of its appealing functionality, FL has been shown vulnerable to some attacks. For example, the works [4], [5], [6] show that the gradient vector uploaded by a client may leak sensitive information about its private dataset. To address

X. Guo, Z. Liu, J. Gao and B. Hou are with the College of Cyber Science, College of Computer Science, Tianjin Key Laboratory of Network and Data Security Technology, Nankai University, China. Email: xiaojieg.veda@gmail.com, liuzheli@nankai.edu.cn,pdsgjq@163.com, houboyu@dbis.nankai.edu.cn.

$\mathrm{J}$. Li is with the School of Computer Science, Guangzhou University, China, also with the College of Cyber Science, Nankai University, China. Email: jinli71@gmail.com.

C. Dong is with School of Computing, Newcastle University, Newcastle upon Tyne, U.K. Email: changyu.dong@ncl.ac.uk.

T. Baker is with the Liverpool John Moores University, United Kingdom. Email: t.baker@ljmu.ac.uk.

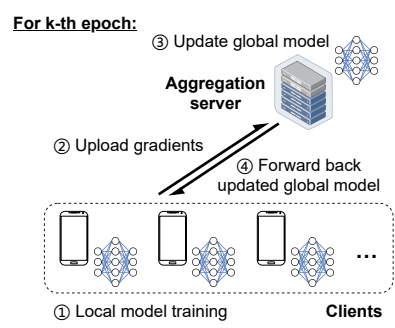

(a)

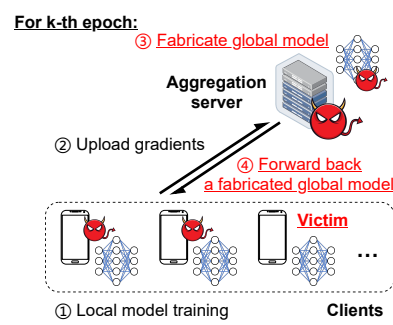

(b)
Fig. 1. Federated learning and a trivial attack on it. (a) The framework of federated learning. (b) The attack launched by the adversary corrupting the server and a subset of clients.

this issue, in [7], the authors proposed a secure aggregation protocol that guarantees the privacy of gradients. However, the recent work [8] shows that, in addition to the gradient privacy, the integrity of aggregation should be protected as well. In particular, the server can easily become a single-point of failure in FL. Without integrity guarantee, once the server gets compromised, the adversary controlling the server can manipulate the global model and cause misclassification of any involved client at its specified data point (Figure 1(b)), which is similar to the consequence of backdoor attacks [9], [10], [11], [12]. The lack of the integrity guarantee of aggregation may restrict the commercial application of FL. An example attack is that the corrupted server re-trains the global model updated in this epoch with some poisoned data and returns the re-trained model to honest clients, aiming to cause misclassification in these clients.

We note that attacks by modifying aggregation results (e.g., the aforementioned example attack) can be mitigated using verifiable aggregation protocols. In such protocols for FL, a considered adversary cannot convince an honest client to accept its forged aggregated gradient as a real one with an overwhelming probability (see Definition 3). Certainly, to resist inference attacks exploiting the "non-encrypted" gradients [4], [5], [6], the used verifiable aggregation protocol should be secure as well to guarantee input privacy of each client. Informally speaking, this means that an adversary in the aggregation protocol learns nothing about the gradient of each client from protocol transcripts (see Definition 4).

In this paper, we focus on how to realize a (secure) verifiable aggregation protocol in a communication-efficient and fast way on resource-constrained devices (e.g., the setting of mobile devices in Google's work [7]). Since the bandwidth in the resource-constrained setting is expensive and a large number of clients are required to train a global model through 
hundreds of iterations, communication becomes one of the most important considerations in the design of FL protocol. If the designed verifiable aggregation protocol costs a lot in terms of communication and computation, it will take a long period to finish the training process. There are many factors influencing the communication efficiency of a verifiable aggregation protocol, and the number of parameters in the model to be trained is particularly important. So, our first question is, can we design a verifiable aggregation protocol in which the communication of verification is independent of the number of parameters in the model to be trained? In fact, the communication efficiency of FL is always a research hotspot [1], [7], [2], [13].

To the best of our knowledge, there is no previous work that succeeds in designing a communication-efficient verifiable aggregation protocol. The works about communicationefficient FL [7], [13] do not consider how to guarantee the integrity of aggregation. The recent work that achieves the first secure verifiable aggregation [8] uses zero-knowledge proof to enforce honest aggregation. However, its communication cost for verification is linearly dependent on the dimension of gradient vectors. Its linearly-growing and high communication cost makes it impractical in its intended application scenarios, that is FL among mobile devices. Moreover, its computation cost for verification is unsatisfactory due to the use of zero-knowledge. For example, it takes $3.59 \mathrm{MB}$ outgoing communication and $254964 \mathrm{~ms}$ computation per client to verify the aggregation of $20 \mathrm{~K}$-dimensional parameter vectors among 500 clients. Another trivial solution to achieve verifiable aggregation is using generic multiparty computation (MPC) approaches [14], [15], [16], [17], [18], [19], [20], [21], but its communication and computational overhead are very large. So, in addition to communication efficiency, our second question is, how to make our verifiable aggregation protocol computation-efficient?

\section{A. Challenge and Contribution}

Challenge. To verify the integrity of aggregation, a straightforward idea is to use homomorphic hash to compute the "digest" of gradient vectors to be aggregated. A combined hash can be obtained from these hash values after aggregation, and each client compares it with the hash of the aggregation result (i.e., real hash) to verify the aggregation. Although this idea yields a desirable feature that the verification communication is independent of gradient dimension, we would like to show that this idea provides little security guarantee.

The security challenge comes from the fact that everyone in the above construction has access to the homomorphic hash function. In the simulation-based proof, the simulator did not know the input (i.e., the gradient vector) of each honest client by the time it was asked to simulate its hash value. To simulate it, the simulator has to use a dummy vector. However, this dummy value is different from the real one with an overwhelming probability. Since everyone (including the adversary) can call the homomorphic hash function with the aggregation result, an inconsistency between the combined and real hashes will be found after aggregation and this immediately yields an efficient distinguisher that fails the simulation. That is, the straightforward construction is not secure.

In [8], the authors solve this security challenge by combining homomorphic hash with zero-knowledge proof. However, due to the dimension-wise zero-knowledge proofs, their protocol leads to expensive computation and the communication caused by verification is still linear in the gradient dimension. We note that such dimension-dependence is depressing since the gradient dimension is large in real-world FL applications (e.g., 100K). We are motivated to find another way to achieve verifiable aggregation, overcoming the security challenge with good concrete efficiency in terms of communication and computation.

Contribution. In this paper, we present a communicationefficient and fast protocol, VERIFL, for verifiable aggregation in FL. As shown by experiment, for example, VERIFL achieves $33.24 \mathrm{~KB}$ (dimension-independent) outgoing communication and $8899 \mathrm{~ms}$ computation per client to verify the aggregation of $100 \mathrm{~K}$-dimensional parameter vectors among 500 clients, which outperforms the state-of-the-art work [8] by $110.6 \times$ in communication and $28.7 \times$ in computation even with $5 \times$ larger parameter vectors. More specifically, we make the following contributions:

- (Secure) verifiable aggregation. To verify the integrity of aggregation, we combine the linearly homomorphic hash with the commitment scheme to force the aggregation server to use the submitted gradients that are consistent with their previously broadcast hashes. Collision resistance of the hash scheme guarantees that the server cannot have an honest client accept its forged result. In addition, to overcome the aforementioned security issue, we observe that it sufficient to use equivocal commitment scheme to achieve the same security as [8] without using heavy zero-knowledge proof. Based on this observation, we develop a novel verifiable aggregation protocol. Notably, secure aggregation is also considered in our protocol and we show our protocol is composable with the secure protocol in [7] by using double-masking technique and adding extra rounds. The functionality of secure aggregation in our protocol is achieved in the sense that only the aggregation result computed by the server is revealed.

- Dimension-independent communication overhead. In our protocol, the communication overhead resulted from integrity verification is independent of the dimension of gradient vectors (i.e., the number of parameters in FL models), which significantly saves the bandwidth of clients. This is achieved by asking each client to commit only the hash of its gradient vector instead of the vector itself. These commitment strings, which facilitate our security proof, have a length independent of the gradient dimension. The linear homomorphism of the hash scheme ensures that the "sum" of hashes equals to the hash of the sum vector. It is helpful to think that linearly homomorphic hash can be used to compress high-dimensional gradient vectors while preserves the properties of addition. 
- Approximately-halved computation overhead. We succeed in reducing the verification cost of our basic construction in terms of computation by using amortized verification. In our protocol, the most time-consuming operation is the two calls of linearly homomorphic hash that requires $O(d)$ expensive modular exponentiations, in which $d$ is the dimension of gradient vectors. The former call is used to generate the necessary messages for future verification and is inevitable. However, the latter call that performs the integrity verification can be amortized. First, we draw a set of random coefficients to compute the linear combination of the hash aggregations in different epochs. Then, we check whether the combined hash is equal to the hash of the linear combination (using the same coefficients) of aggregation results in different epochs. In this way, we only performs the integrity verification once a batch and the cost of the latter call is amortized by a factor of the batch size. The total computation overhead can be approximately reduced to that of the former hash call.

\section{Federated Learning with Secure Aggregation}

\section{A. Federated Learning}

The basic framework of FL is summarized as follows. There are $N$ users in FL, each having access to its private dataset $\mathcal{D}_{i}$ where $\left|\mathcal{D}_{i}\right|=s_{i}$. At the beginning of each epoch $k \in\{1,2, \ldots\}$, the aggregation server will randomly select a subset of users $S_{k}$ and send them the parameter vector $\mathbf{v}\langle k-1\rangle$ obtained in the previous epoch (note that $\mathbf{v}\langle 0\rangle$ is initialized randomly). Each selected user $\mathcal{P}_{i}$ will locally minimize the empirical loss over its dataset $\mathcal{D}_{i}$ to get its updated (local) model $\mathbf{v}_{i}\langle k\rangle$ and upload its gradient $\Delta_{i}\langle k\rangle \leftarrow \mathbf{v}_{i}\langle k\rangle-\mathbf{v}_{i}\langle k-1\rangle$ to the aggregation server. Upon receiving enough gradients from the users, typically, the server will take a weighted average of these gradients and obtain an updated global model, i.e., $\mathbf{v}\langle k\rangle \leftarrow \mathbf{v}\langle k-1\rangle+\sum_{i \in S_{k}} \beta_{i} \Delta_{i}\langle k\rangle$ where $\beta_{i}=s_{i} / \sum_{i \in S_{k}} s_{i}$. The training process will continue until model convergence.

\section{B. Secure Aggregation}

In [7], the authors build up a secure and dropout-tolerant aggregation protocol based on double-masking technique. More specifically, the double-masking of a gradient vector $\mathbf{v}_{i}$ includes two parts: the self-mask generated by the owner $\mathcal{P}_{i}$ itself, and the pairwise-mask generated between $\mathcal{P}_{i}$ and each other client. The double-masked gradient of $\mathbf{v}_{i}$ is denoted by $\mathbf{p}_{i}$ :

$$
\begin{aligned}
\mathbf{p}_{i} & =\mathbf{v}_{i}+\underbrace{\operatorname{PRG}\left(b_{i}\right)}_{\text {self }- \text { mask }} \\
& +\underbrace{\sum_{j \in \mathcal{U}, i<j} \operatorname{PRG}\left(\operatorname{mak}_{i, j}\right)-\sum_{j \in \mathcal{U}, i>j} \operatorname{PRG}\left(\operatorname{mak}_{i, j}\right)}_{\text {pairwise }- \text { mask }} \bmod B
\end{aligned}
$$

where $B$ is the modulus for aggregation, $b_{i}$ is a secret seed sampled by the $\mathcal{P}_{i}$ and $\mathrm{mak}_{i, j}=\mathrm{mak}_{j, i}$ is a pairwise agreed value between $\mathcal{P}_{i}$ and $\mathcal{P}_{j}$ for each $\mathcal{P}_{j} \in \mathcal{U}$. Rounds of their protocol are briefly summarized in Figure 2.

\section{Secure Aggregation Protocol}

- Round 0 (AdvertiseKeys): Each client $\mathcal{P}_{i}$ generates two key pairs $\left(\mathrm{sk}_{i}, \mathrm{pk}_{i}\right),\left(\mathrm{msk}_{i}, \mathrm{mpk}_{i}\right)$ and sends $\left(\mathrm{pk}_{i}, \mathrm{mpk}_{i}\right)$ to other clients. The server serves as a relay and appends each client that is alive at present to the set $\mathcal{U}_{1}$.

- Round 1 (ShareKeys): Each client $\mathcal{P}_{i}$ samples a secret value $b_{i}$ and generates secret shares of $\left(\mathrm{msk}_{i}, b_{i}\right)$ to be distributed among other clients. For each other client $\mathcal{P}_{j}$, it derives pairwise symmetric encryption key $\mathrm{k}_{i, j}$ from $\mathbf{s k}_{i}$ and $\mathrm{pk}_{j}$ using key agreement. After that, it encrypts the shares for each $\mathcal{P}_{j}$ using $\mathrm{k}_{i, j}$ and sends the ciphertext to $\mathcal{P}_{j}$. The server serves as a relay and appends each client that is alive at present to the set $\mathcal{U}_{2}$.

- Round 2 (MaskedInputCollection): Each client $\mathcal{P}_{i}$ computes its double-masked gradient vector $\mathbf{p}_{i}$, in which the pairwise agreed $\mathbf{m a k}_{i, j}$ is derived from $\mathbf{m s k}_{i}$ and $\mathrm{mpk}_{j}$ using key agreement, and sends $\mathbf{p}_{i}$ to the server. The server appends each client that is alive at present to the set $\mathcal{U}_{3}$.

- Round 3 (Unmasking): The server asks the clients in $\mathcal{U}_{3}$ for: (i) all secret shares of $b_{i}$ 's for $i \in \mathcal{U}_{3}$, and (ii) all secret shares of $\mathrm{msk}_{i}$ for $i \in \mathcal{U}_{2} \backslash \mathcal{U}_{3}$. The aggregation result $\mathbf{a}=\sum_{i \in \mathcal{U}_{3}} \mathbf{p}_{i}-\sum_{i \in \mathcal{U}_{3}} \mathrm{PRG}\left(b_{i}\right)+$ $\sum_{i \in \mathcal{U}_{2} \backslash \mathcal{U}_{3}, j \in \mathcal{U}_{3}} \Delta_{i, j} \operatorname{PRG}\left(\operatorname{mak}_{i, j}\right)$, in which $b_{i}$ is directly derived from share reconstruction, mak $_{i, j}$ is derived from the reconstructed $\mathrm{msk}_{i}$ and the public $\mathrm{mpk}_{j}$, and $\Delta_{i, j}=1$ if $i<j$ and $\Delta_{i, j}=-1$ if $i>j$ and $\Delta_{i, j}=0$ if $i=j$.

Fig. 2. The semi-honest version protocol in [7].

\section{Adversarial Model}

In this paper, we consider the same adversary as in [8]. The adversary is semi-honest but with the additional power to instruct the corrupted server to forge the aggregation result arbitrarily with the knowledge of the transcripts it has seen before. The adversary can corrupt both the aggregation server and a subset of data parties (i.e., clients). In this semihonest model, the corrupted parties will provide their gradients honestly. Using the notations to be introduced in the next section, all gradient vectors of parties belong to $\mathbb{Z}_{R}^{d}$ and the aggregation result should lie in $\mathbb{Z}_{B}^{d}$ for some bound $B \geq N \cdot R$, in which $N$ is the number of parties.

In this adversarial model, the adversary may aim either (i) to infer the private gradients of honest parties, or (ii) to convince honest parties of its forged aggregation results. The goal of our protocol is to protect the privacy of each party's gradient while guarantees the integrity of aggregation. However, we do not consider the adversary that makes queries to the trained model to launch black-box statistical attacks [22], [23], [24], [25], [26] since it is known hard to prevent the leakage from the output of the functionality implemented by cryptographic protocols. Moreover, such attacks might not work well to precisely infer the sensitive information of honest parties, especially for deep neural networks that generalize well [6].

Note that, in FL, the private inputs of honest parties in the previous round may be approximate to the those in the current round when the model converges. To forge an approximate aggregation result, it suffices for the adversary to choose the inputs of corrupted parties according to the partial sum of the inputs of honest parties in the previous round. This observation 
can lead to a model replacement attack in [9], which bypasses secure aggregation protocols and is possible since some small perturbations will not significantly affect the behaviour of machine learning models. How to prevent such an attack in FL settings is another interesting and open problem, which is out of the scope of this paper. In this paper, like other works in the literature of multiparty computation, we only focus on how to prevent the adversary from forging an aggregation result different from the exact one. We do not consider the attacks (e.g., model replacement attack) where the adversary chooses its input according to the past protocol transcripts. This is known challenging in multiparty computation since it cannot be distinguished from the case where a corrupted party do use this as its input without some apriori knowledge of input.

\section{VERIFL: EFFICIENT VERIFIABLE AgGREGATION}

\section{A. Notations}

We use $[n]$ to denote the set $\{1, \ldots, n\}$ for some integer $n$. The set of integers is denoted by $\mathbb{Z}$. The quotient ring of integers modulo a positive integer $q$ is written in $\mathbb{Z}_{q}$. When $q$ is a prime, $\mathbb{Z}_{q}$ is a field denoted by $\mathbb{F}_{q}$. A vector is denoted by a bold lower case letter, e.g., $\mathbf{x}$, and its $i$-th entry is denoted by $\mathbf{x}[i]$ where the index $i$ is counted from one. For some finite set $\mathcal{X}$, its cardinality is $|\mathcal{X}|$. If $\mathcal{A}$ is an algorithm, $a \leftarrow \mathcal{A}$ says that $a$ is assigned to be the output of $\mathcal{A}$; otherwise, if $\mathcal{A}$ is a set, $a$ is an element uniformly drawn from the set $\mathcal{A}$. The security parameter is denoted $\kappa$ and we use negl $(\kappa)$ to denote the negligible function in $\kappa$. We use $\mathcal{A}(x ; y)$ to denote that an algorithm $\mathcal{A}$ is invoked with a public input $x$ and a secret input $y$. We formulate pseudo-random generator as PRG : $\{0,1\}^{*} \mapsto \mathbb{Z}_{B}^{d}$ for some modulus $B$ and vector dimension $d$.

\section{B. Cryptographic Primitives}

Linear homomorphic hash. A linearly homomorphic hash scheme consists of three polynomial-time algorithms, i.e., $\mathrm{LHH}=$ (LHH.HGen, LHH.Hash, LHH.Eval). We detail the above three algorithms as constructed in [27] by assuming the hardness of discrete logarithm.

- LHH.HGen $\left(1^{\kappa}, 1^{d}\right)$ : On input the security parameter $\kappa$ and the dimension $d$, this algorithm outputs the public parameter LHHpp, including the description of a cyclic group $\mathbb{G}$ of prime order $q$, its generator $g \in \mathbb{G}$ and $d$ distinct elements $g_{1}, \ldots, g_{d} \in \mathbb{G}$. For simplicity of presentation, this public parameter will be taken implicitly as the first parameter of LHH.Hash and LHH.Eval.

- LHH.Hash(x): Taking a $d$-dimensional vector $\mathbf{x}$ as input, this algorithm outputs the linearly homomorphic hash of $\mathbf{x}: h \leftarrow \prod_{i \in[d]} g_{i}^{\mathbf{x}}{ }^{[i]} \in \mathbb{G}$.

- LHH.Eval $\left(h_{1}, \ldots, h_{\ell}, \alpha_{1}, \ldots, \alpha_{\ell}\right)$ : Taking $\ell$ hashes and $\ell$ coefficients of linear combination, this algorithm outputs the linear combination of these $\ell$ hashes: $h^{*} \leftarrow$ $\prod_{i \in[\ell]} h_{i}^{\alpha_{i}}$.

Note that this construction of LHH satisfies the following definition of collision resistance with respect to the collision experiment $\operatorname{Expt}_{\mathcal{A}, \mathrm{LH} H}^{\text {coll }}$.

$$
\begin{aligned}
& \text { Expt } \operatorname{Ex}_{\mathcal{A}, \mathrm{LHH}}^{\text {coll }}\left(1^{\kappa}, 1^{d}\right) \text { : } \\
& \text { 1) Call LHHpp } \leftarrow \text { LHH.HGen }\left(1^{\kappa}, 1^{d}\right) \text {. } \\
& \text { 2) Send LHHpp to the adversary and wait for its input } \\
& \left(\mathbf{x}_{1}, \mathbf{x}_{2}\right) \leftarrow \mathcal{A}\left(\text { LHHpp) where } \mathbf{x}_{1}, \mathbf{x}_{2} \in \mathbb{F}_{q}^{d}\right. \text { are two } \\
& \text { distinct vectors. } \\
& \text { 3) Output } 1 \text { iff LHH.Hash }\left(\mathbf{x}_{1}\right)=\mathrm{LHH} . \operatorname{Hash}\left(\mathbf{x}_{2}\right) ; \\
& \text { otherwise output } 0 .
\end{aligned}
$$

Definition 1 (Collision resistance). $L H H$ is said to be collision-resistant, if for any PPT adversary $\mathcal{A}$, there exists a negligible function negl(.) such that the advantage of $\mathcal{A}$

$$
A d v_{\mathcal{A}, L H H}^{\text {oll }}(\kappa):=\operatorname{Pr}\left[\operatorname{Expt}_{\mathcal{A}, L H H}^{\text {coll }}\left(1^{\kappa}, 1^{d}\right)=1\right] \leq \operatorname{negl}(\kappa)
$$

for the security parameter $\kappa$ and the vector dimension $d$.

Commitment. Commitment is an "envelope" so that a party cannot change the value after they have committed to it while the committed value is kept secret to others before decommitment. The commitment scheme used in this paper is to make the security go through without leading to too much communication overhead for verification. In particular, to formally prove the security of our protocol, we need an equivocal commitment scheme. Roughly speaking, someone possessing the trapdoor of an equivocal commitment scheme can produce commitments that can be opened to different values. However, committers in real world, who have no idea about the trapdoor, can open them to only a single value. Such property enables the simulator in our proof to fool the distinguisher and achieves indistinguishability consequently (see Section IV).

A non-interactive equivocal commitment scheme is defined as a tuple of four polynomial-time algorithms COM = (COM.Setup, COM.Commit, COM.Decommit, COM.Equiv):

- COM.Setup $\left(1^{\kappa}\right)$ : On input the security parameter $\kappa$, this algorithm outputs a public parameter COMpp and a trapdoor td. Note that the message space $\mathcal{M}$ and the commitment space $\mathcal{C}$ are also provided in COMpp. For simplicity of presentation, this public parameter will be taken implicitly as the first parameter of the other algorithms.

- COM.Commit $(m ; r)$ : This algorithm is run by the committer and takes as input a message to be committed $m \in \mathcal{M}$ and a uniform randomness $r$ and outputs a commitment string $c \in \mathcal{C}$, which is to be publicly published. Note that the randomness $r$ serves as the "decommitment string" to open the committed message and it should be kept secret until opening.

- COM.Decommit $\left(c, m^{\prime}, r^{\prime}\right)$ : This algorithm is run by the receiver and takes as input a commitment string $c \in \mathcal{C}$, a claimed committed message $m^{\prime} \in \mathcal{M}$ and the claimed randomness $r^{\prime}$ that was used to commit $m^{\prime}$. If $c=$ COM.Commit $\left(m^{\prime} ; r^{\prime}\right)$ then output 1 ; otherwise output 0 .

- COM.Equiv $\left(c,(m, r), m^{\prime}\right.$; td $)$ : This algorithm takes as input a commitment string $c \leftarrow \operatorname{COM}$.Commit $(m ; r) \in$ $\mathcal{C}$, a desired arbitrary message $m^{\prime} \in \mathcal{M}$ and the trapdoor 
td and outputs an valid randomness $r^{\prime}$ such that $c$ decommits to $m^{\prime}$.

The equivocality of COM is formally defined as follows.

Definition 2 (Equivocality). COM is said to be equivocal if for any $m \in \mathcal{M}$ and uniform randomness $r$, it holds that there exists a negligible function negl(.) such that

$$
\begin{aligned}
& \operatorname{Pr}\left[\begin{array}{l|l}
b=0 & \left.\begin{array}{l}
(\text { COMpp, td }) \leftarrow \text { COM.Setup }\left(1^{\kappa}\right), \\
c \leftarrow \text { COM.Commit }(m ; r), \\
m^{\prime} \leftarrow \mathcal{M}, \\
r^{\prime} \leftarrow \text { COM.Equiv }\left(c,(m, r), m^{\prime} ; t d\right) \\
b \leftarrow \text { COM.Decommit }\left(c, m^{\prime}, r^{\prime}\right)
\end{array}\right]
\end{array}\right. \\
& \leq \operatorname{negl}(\kappa)
\end{aligned}
$$

for the security parameter $\kappa$.

\section{Symmetric Encryption. An symmetric encryp-} tion scheme is defined to be a tuple $\mathrm{SE}=$ (SE.KeyGen, SE.Enc, SE.Dec).

- SE.KeyGen $\left(1^{\kappa}\right)$ : On input the security parameter $\kappa$, this algorithm outputs a secret symmetric key k.

- SE.Enc $(m ; \mathbf{k})$ : This algorithm takes as input a message $m$ to be encrypted and a symmetric key k. The output of this algorithm is the ciphertext $c$ of $m$ under the key $\mathrm{k}$.

- $\mathrm{SE} . \operatorname{Dec}\left(c ; \mathrm{k}^{\prime}\right)$ : This algorithm takes as input a ciphertext to be decrypted and its corresponding symmetric key $\mathrm{k}^{\prime}$. The output of this algorithm is the message $m^{\prime}$ such that $c \leftarrow \operatorname{SE} . \operatorname{Enc}\left(m^{\prime} ; \mathbf{k}^{\prime}\right)$.

In this paper, we require SE to be IND-CPA secure.

Key Agreement. We use a key agreement scheme to (i) generate pairwise symmetric encryption keys, and (ii) generate pariwise seeds for PRG. A key agreement scheme is defined to be a tuple $\mathrm{KA}=(\mathrm{KA}$.Setup, KA.KeyGen, KA.Agree $)$.

- KA.Setup $\left(1^{\kappa}\right)$ : On input the security parameter $\kappa$, this algorithm outputs a public parameter KApp. For simplicity of presentation, this public parameter will be taken implicitly as the first parameter of the other algorithms.

- KA.KeyGen(): This algorithm generates a key pair (sk, pk).

- KA.Agree $\left(\mathrm{sk}_{i}, \mathrm{pk}_{j}\right)$ : This algorithm takes as input a secret key $\mathrm{sk}_{i}$ and a public key $\mathrm{pk}_{j}$ and outputs a private agreed key $\mathrm{ak}_{i, j}$.

Secret Sharing. We use secret sharing to deal with dropout in our protocol and preserve input privacy. A secret sharing scheme is defined to be a tuple SS= (SS.Setup, SS.Share, SS.Combine).

- SS.Setup $\left(1^{\kappa}\right)$ : On input the security parameter $\kappa$, this algorithm outputs a public parameter SSpp, which includes the message space $\mathcal{M}$. For simplicity of presentation, this public parameter will be taken implicitly as the first parameter of the other algorithms.

- SS.Share $(t, \mathcal{P}, s)$ : This algorithms takes as input the threshold value $t$, the set of parties $\mathcal{P}$ which is of size $N \geq t$ and a secret $s \in \mathcal{M}$. The output of this algorithm is a set of secret shares, denoted by $\left\{\llbracket s \rrbracket_{i}\right\}_{\mathcal{P}_{i} \in \mathcal{P}}$, each of which is assigned to a distinct holder $\mathcal{P}_{i} \in \mathcal{P}$.

- SS.Combine $\left(t,\left\{\llbracket s \rrbracket_{i}\right\}_{\mathcal{P}_{i} \in \mathcal{P}^{\prime} \subseteq \mathcal{P}}\right)$ : This algorithm takes as input the threshold value $t$ and the subset of shares $\left\{\llbracket s \rrbracket_{i}\right\}_{\mathcal{P}_{i} \in \mathcal{P}^{\prime} \subseteq \mathcal{P}}$ of which the size is not less than $t$. The output of this algorithm is the original secret $s$.

\section{How to Achieve Efficient Verifiable Aggregation}

Basic verifiable aggregation. The integrity of aggregation is achieved by adding the following two steps to the basic FL framework:

PREPARATION: This step is done by clients before they submit their gradient vectors to the server. In this step, each client is asked to generate: (i) the linearly homomorphic hash of its gradient vector, and (ii) the commitment string of this hash value. More formally, $\mathcal{P}_{i}$ generates

$$
\begin{aligned}
h_{i} & \leftarrow \operatorname{LHH} . \operatorname{Hash}\left(\mathbf{v}_{i}\right), \\
c_{i} & \leftarrow \operatorname{COM} . \operatorname{Commit}\left(h_{i} ; r_{i}\right),
\end{aligned}
$$

in which $h_{i}$ is the linearly homomorphic hash of $\mathbf{v}_{i}, c_{i}$ is the commitment string and $r_{i}$ is a uniformly random string secretly sampled by $\mathcal{P}_{i}$. Note that $\left(h_{i}, r_{i}\right)$ serves as the decommitment string of $\mathcal{P}_{i}$. Note that, after this step, the commitment string $c_{i}$ will be forwarded to other clients. $\mathcal{P}_{i}$ will not send its gradient vector to the server until it receives from all other clients their $c_{i}$ 's respectively.

VERIFICATION: This step is done by each client after it receives from the server the aggregation result $\mathbf{a}$. In this step, $\mathcal{P}_{i}$ asks each other client $\mathcal{P}_{j}$ for its decommitment string $\left(h_{j}, r_{j}\right)$ and checks whether

$$
1 \stackrel{?}{=} \text { COM.Decommit }\left(c_{j}, h_{j}, r_{j}\right),
$$

in which $c_{i}$ was received after PREPARATION step. If the equality test (1) does not hold for some $j \in[N] \backslash\{i\}$, then the aggregation result a will be regarded as forged and $\mathcal{P}_{i}$ terminates with output $\perp$. Otherwise $\mathcal{P}_{i}$ will continue to check the equality of hashes:

$$
\operatorname{LHH} . \operatorname{Hash}(\mathbf{a}) \stackrel{?}{=} \operatorname{LHH} . \operatorname{Eval}(h_{1}, \ldots, h_{N}, \underbrace{1, \ldots, 1}_{\mathrm{N} \text { items }}) .
$$

If the equality test (2) holds, then the aggregation result a passes the verification and $\mathcal{P}_{i}$ will accept the result; otherwise the result will be regarded as forged and $\mathcal{P}_{i}$ terminates with output $\perp$.

Dimension-independent communication overhead. The reduction in the communication overhead for verification comes from the usage of hash and commitment. Recall that the outgoing message for verification sent by each client $\mathcal{P}_{i}$ in each epoch consists of the commitment string $c_{i}$ and the decommitment string $\left(h_{i}, r_{i}\right)$. As long as we carefully instantiate COM with the one using commitment/decommitment strings of constant length, the outgoing communication for verification is constant and independent of the the dimension of gradient vectors. That is, in each epoch, the outgoing communication cost lead by verification can be made only $O(1)$ and the incoming communication cost is $O(N)$.

Approximately-halved computation overhead. In our protocol, the most time-consuming operation for verification is running LHH.Hash. To reduce the computation overhead for verification, we allow each client to do verification in an amortized manner. Similar to [14], [15], [28], we draw a set 
of random coefficients to compute the linear combination of the hash aggregations in different epochs. Then, we check whether the combined hash is equal to the hash of the linear combination (using the same coefficients) of aggregation results in different epochs. More formally, letting $\ell$ be the preset batch size and $\mathbf{a}\langle k\rangle$ be the aggregation result in the epoch $k \in[\ell]$, we replace the aforementioned VERIFICATION step with the following AMORTIZED-VERIFICATION step:

AMORTIZED-VERIFICATION: The equality test of commitment (1) remains unchanged for all commitments received in each epoch $k \in[\ell]$. However, for $k \in[\ell], \mathcal{P}_{i}$ does the following:

$$
\begin{aligned}
\alpha_{k} & \leftarrow \mathbb{F}_{q}, \\
h\langle k\rangle & \leftarrow \operatorname{LHH} . \operatorname{Eval}(h_{1}\langle k\rangle, \ldots, h_{N}\langle k\rangle, \underbrace{1, \ldots, 1}_{\mathrm{N} \text { items }}),
\end{aligned}
$$

in which $q$ is the prime order provided in LHHpp and $h_{i}\langle k\rangle$ is the hash computed by client $\mathcal{P}_{i}$ in the $k$-th epoch. Then, it verifies the equality

$$
\begin{aligned}
& \operatorname{LHH} . \text { Hash }\left(\sum_{k \in[\ell]} \alpha_{k} \mathbf{a}\langle k\rangle\right) \\
& \stackrel{?}{=} \operatorname{LHH} . \operatorname{Eval}\left(h\langle 1\rangle, \ldots, h\langle\ell\rangle, \alpha_{1}, \ldots, \alpha_{\ell}\right)
\end{aligned}
$$

instead of the equality (2). If the equality test (3) holds, then all the aggregation results $\mathbf{a}\langle 1\rangle, \ldots, \mathbf{a}\langle\ell\rangle$ pass the verification and $\mathcal{P}_{i}$ will accept these results; otherwise these results will be regarded as forged and $\mathcal{P}_{i}$ terminates with output $\perp$.

In this way, $\mathcal{P}_{i}$ does not need to call LHH.Hash in the equality test (2) every aggregation epoch. That is, the cost for LHH.Hash in the equality test (2) can be amortized by a factor $\ell$. Since the cost for LHH.Hash is $O(d)$, the amortized computation cost for verification in each epoch can be reduced from $O(2 d)$ to $O(d+d / \ell)$. When $\ell$ is large enough, the cost can be approximately halved.

Privacy concern and dropout-tolerance. Our verifiable aggregation protocol (i.e., the basic FL framework with PREPARATION and AMORTIZED-VERIFICATION steps) is composable with the secure one Figure 2. The summarized secure verifiable aggregation protocol is given in Figure 3 . We modify the ShareKeys round in [7] and the original secure protocol serves as the aggregation phase in this composite protocol. Notably, the dropout-tolerance of [7] is preserved and therefore the aggregation phase in our protocol is robust to client dropout. However, to deal with the potential dropout when clients report their decommitment strings in Decommitting round, an extra DroppedDecommitting round is introduced. This extra round guarantees that all clients that survive dropout are able to run AMORTIZED-VERIFICATION in BatchChecking round. Note that, to tolerate dropout, we require the additional $O(N)$ computation of secret sharing in ShareMetadata round. Therefore, the total verification cost in computation is $O(N+d)$.

\section{Full Version of VERIFL Protocol}

The full version of VERIFL Protocol is presented in Figure 4 and Figure 5, where cryptographic primitives are defined in Section III-B.

\section{VERIFL protocol}

1) Aggregation phase (for the epoch $k \in[\ell]$ ):

- Round 0 (AdvertiseKeys): Identical to Round 0 in Figure 2 with additional setups of $\mathrm{LHH}$ and COM.

- Round 1 (ShareMetadata): Identical to Round 1 in Figure 2 , except that each client $\mathcal{P}_{i}$ (i) additionally runs the PREPARATION step to get its $c_{i}\langle k\rangle$ and $\left(h_{i}\langle k\rangle, r_{i}\langle k\rangle\right)$, (ii) shares and encrypts $\left(h_{i}\langle k\rangle, r_{i}\langle k\rangle, \mathrm{msk}_{i}\langle k\rangle, b_{i}\langle k\rangle\right)$ instead of $\left(\mathrm{msk}_{i}\langle k\rangle, b_{i}\langle k\rangle\right)$, and (iii) sends $c_{i}\langle k\rangle$ to each other client along with the ciphertext.

- Round 2 (MaskedInputCollection): Identical to Round 2 in Figure 2.

- Round 3 (Unmasking): Identical to Round 3 in Figure 2, except that the server explicitly sends the aggregation result $\mathbf{a}\langle k\rangle$ to each client.

2) Verification phase (of batch size $\ell$ ):

- Round 0 (Decommitting): For each $k \in[\ell]$, each client $\mathcal{P}_{i}$ sends its decommitment strings $\left(h_{i}\langle k\rangle, r_{i}\langle k\rangle\right)$ to each other client using the server as a relay. The server appends each client that is alive at present to the set $\mathcal{V}_{1}$.

- Round 1 (DroppedDecommitting): For each $k \in[\ell]$, each client $\mathcal{P}_{i}$ sends its shares of $h_{j}\langle k\rangle$ and $r_{j}\langle k\rangle$ to the server, in which $j \in \mathcal{U}_{3}\langle k\rangle \backslash \mathcal{V}_{1}$. The server reconstructs these $\left(h_{j}\langle k\rangle, r_{j}\langle k\rangle\right)$ 's and sends them to each alive client.

- Round 2 (BatchChecking): Each client $\mathcal{P}_{i}$ runs the AMORTIZED-VERIFICATION step.

Fig. 3. Our verifiable aggregation protocol with secure aggregation functionality.

\section{Security Analysis}

In this section, we will show that our protocol ensures the integrity of the aggregation results (Definition 3 ) and the privacy of individual input (Definition 4). Recall that our protocol runs with a set $\mathcal{P}$ of $N$ parties and an aggregation server $\mathcal{S}$, in which the building blocks are instantiated with the security parameter $\kappa$ and the verification is performed in batch of size $\ell$. The threshold for dropout is $t$ (i.e., at least $t$ parties survive dropout) and the set of corrupted parties is $\mathcal{C} \subseteq \mathcal{P} \cap\{\mathcal{S}\}$ such that $|\mathcal{C} \backslash\{\mathcal{S}\}|<t . \mathcal{U}_{*}\langle k\rangle$ is the set $\mathcal{U}_{*}$ in the $k$-th aggregation phase. Given fixed $N, t, \kappa, \ell$ and $\mathcal{C}$, we define $M_{\mathcal{C}}$ as the polynomial time algorithm for the "nextmessage" function of corrupted parties in $\mathcal{C}$. That is, given a party identifier $c \in \mathcal{C}$, a round index $i$ a transcript $T_{i}$ that has been sent and received so far by all corrupted parties in $\mathcal{C}$, and the joint randomness $r_{\mathcal{C}}$ for the execution of corrupted parties, $M_{\mathcal{C}}\left(c, i, T_{i}, r_{\mathcal{C}}\right)$ outputs the message for the party $c$ in the round $i$.

Note that some common reference strings (CRS) are required by this protocol. As we will see, this protocol is secure in the CRS-hybrid model [29]. More specifically, according to the CRS functionality, the simulator is allowed to learn the trapdoor of the underlying commitment scheme. Therefore, in the CRS-hybrid model, the simulator of our protocol can obtain this trapdoor by simulating the CRS functionality and then use the trapdoor to make the simulated view consistent with the real one.

Definition 3 gives out the integrity of aggregation considered in this paper. In this definition, we say that integrity is 
Protocol $\Pi_{V E R I F L}$

1) Common reference string: All parties are given LHHpp $\leftarrow$ LHH.HGen $\left(1^{\kappa}, 1^{d}\right)$, the COMpp output by COM.Setup $\left(1^{\kappa}\right)$, SSpp $\leftarrow \operatorname{SS} . \operatorname{Setup}\left(1^{\kappa}\right)$, and $\mathrm{KApp} \leftarrow \mathrm{KA}$.Setup $\left(1^{\kappa}\right)$. The security parameter $\kappa$, the threshold $t$, the number of parties $N$, the input domain $\mathbb{Z}_{R}^{d}$, the aggregation domain $\mathbb{Z}_{B}^{d}$ and the batch size $\ell$ are implicit.

2) Aggregation phase (for the epoch $k \in[\ell]$ ):

- Round 0 (AdvertiseKeys):

For each party $\mathcal{P}_{i}$ in parallel:

- Generate $\left(\mathrm{sk}_{i}\langle k\rangle, \mathrm{pk}_{i}\langle k\rangle\right) \leftarrow \mathrm{KA} \cdot \operatorname{KeyGen}(),\left(\mathrm{msk}_{i}\langle k\rangle, \mathrm{mpk}_{i}\langle k\rangle\right) \leftarrow \mathrm{KA} \cdot \operatorname{KeyGen}()$.

- Send $\left(\mathrm{pk}_{i}\langle k\rangle, \mathrm{mpk}_{i}\langle k\rangle\right)$ to the server and move to the next round.

Server:

- Collect messages from at least $t$ parties in the previous round; otherwise abort. Let $\mathcal{U}_{1}\langle k\rangle$ be this set of parties.

- Multi-send to all parties in $\mathcal{U}_{1}\langle k\rangle$ the set $\left\{\left(j, \mathrm{pk}_{j}\langle k\rangle, \mathrm{mpk}_{j}\langle k\rangle\right)\right\}_{j \in \mathcal{U}_{1}\langle k\rangle}$ and move to the next round.

- Round 1 (ShareMetadata):

For each party $\mathcal{P}_{i}$ in parallel:

- Receive the set $\left\{\left(j, \mathrm{pk}_{j}\langle k\rangle, \mathrm{mpk}_{j}\langle k\rangle\right)\right\}_{j \in \mathcal{U}_{1}\langle k\rangle}$ from the server. Assert that $\left|\mathcal{U}_{1}\langle k\rangle\right| \geq t$, that all the public keys are different.

- Compute the linearly homomorphic hash of its input in the $k$-th epoch, say $\mathbf{v}_{i}\langle k\rangle$, as well as the commitment of this hash: $h_{i}\langle k\rangle \leftarrow \mathrm{LHH}$.Hash $\left(\mathbf{v}_{i}\langle k\rangle\right)$, $c_{i}\langle k\rangle \leftarrow$ COM.Commit $\left(h_{i}\langle k\rangle ; r_{i}\langle k\rangle\right)$, in which $r_{i}\langle k\rangle$ is uniformly drawn from randomness space.

- Generate $t$-out-of- $\left(\left|\mathcal{U}_{1}\langle k\rangle\right|-1\right)$ shares of $h_{i}\langle k\rangle:\left\{\llbracket h_{i}\langle k\rangle \rrbracket_{j}\right\}_{j \in \mathcal{U}_{1}\langle k\rangle \backslash\{i\}} \leftarrow$ SS.Share $\left(t, \mathcal{U}_{1}\langle k\rangle \backslash\{i\}, h_{i}\langle k\rangle\right)$.

- Generate $t$-out-of- $\left(\left|\mathcal{U}_{1}\langle k\rangle\right|-1\right)$ shares of $r_{i}\langle k\rangle:\left\{\llbracket r_{i}\langle k\rangle \rrbracket_{j}\right\}_{j \in \mathcal{U}_{1}\langle k\rangle \backslash\{i\}} \leftarrow$ SS.Share $\left(t, \mathcal{U}_{1}\langle k\rangle \backslash\{i\}, r_{i}\langle k\rangle\right)$.

- Sample a random element $b_{i}\langle k\rangle \leftarrow \mathbb{F}_{p}$, in which the field $\mathbb{F}_{p}$ is described in SSpp.

- Generate $t$-out-of- $\left(\left|\mathcal{U}_{1}\langle k\rangle\right|-1\right)$ shares of $b_{i}\langle k\rangle:\left\{\llbracket b_{i}\langle k\rangle \rrbracket_{j}\right\}_{j \in \mathcal{U}_{1}}\langle k\rangle \backslash\{i\} \leftarrow$ SS.Share $\left(t, \mathcal{U}_{1}\langle k\rangle \backslash\{i\}, b_{i}\langle k\rangle\right)$.

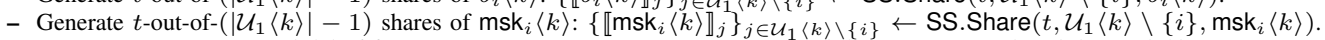

- For each other $\mathcal{P}_{j} \in \mathcal{U}_{1}\langle k\rangle \backslash\{i\}$, compute the pairwise symmetric key $\mathrm{k}_{i, j}\langle k\rangle \leftarrow \mathrm{KA}$.Agree $\left(\mathrm{sk}_{i}\langle k\rangle\right.$, pk $\left.{ }_{j}\langle k\rangle\right)$, and the ciphertext $C_{i, j}\langle k\rangle \leftarrow \operatorname{SE} . \operatorname{Enc}\left(\left(i, j, \llbracket h_{i}\langle k\rangle \rrbracket_{j}, \llbracket r_{i}\langle k\rangle \rrbracket_{j}, \llbracket b_{i}\langle k\rangle \rrbracket_{j}, \llbracket \mathrm{msk}_{i}\langle k\rangle \rrbracket_{j}\right) ; \mathrm{k}_{i, j}\langle k\rangle\right)$.

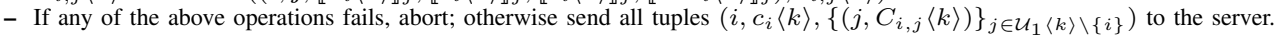

- Store all messages received and values generated in this round, and move to the next round at the end of the message delivery. Server:

- Collect sets of ciphertexts from at least $t$ parties. Let $\mathcal{U}_{2}\langle k\rangle \subseteq \mathcal{U}_{1}\langle k\rangle$ be this set of parties. Send to each party $\mathcal{P}_{j} \in \mathcal{U}_{2}\langle k\rangle$ all message for it: $\left\{\left(i, c_{i}\langle k\rangle, C_{i, j}\langle k\rangle\right)\right\}_{i \in \mathcal{U}_{2}\langle k\rangle \backslash\{j\}}$ and move to the next round.

- Round 2 (MaskedInputCollection):

For each party $\mathcal{P}_{i}$ in parallel:

- Receive from the server the set of messages $\left\{\left(j, c_{j}\langle k\rangle, C_{j, i}\langle k\rangle\right)\right\}_{j \in \mathcal{U}_{2}\langle k\rangle \backslash\{i\}}$ and infer the set $\mathcal{U}_{2}\langle k\rangle$. If the set is of size $\langle t$, abort.

- For each other party $\mathcal{P}_{j} \in \mathcal{U}_{2}\langle k\rangle \backslash\{i\}$, compute the agreed key for pairwise masking $\operatorname{mak}_{i, j}\langle k\rangle=\operatorname{KA}_{\text {. Agree }}\left(\mathrm{msk}_{i}\langle k\rangle, \mathrm{mpk}_{j}\langle k\rangle\right)$.

- Compute its masked input $\mathbf{p}_{i}\langle k\rangle \leftarrow \mathbf{v}_{i}\langle k\rangle+\operatorname{PRG}\left(b_{i}\langle k\rangle\right)+\sum_{j \in \mathcal{U}_{2}\langle k\rangle} \Delta_{i, j} \operatorname{PRG}\left(\operatorname{mak}_{i, j}\langle k\rangle\right) \bmod B$, in which $\Delta_{i, j}=1$ if $i<j$ and $\Delta_{i, j}=-1$ if $i>j$ and $\Delta_{i, j}=0$ if $i=j$.

- If any of the above operations fails, abort; otherwise send $\mathbf{p}_{i}\langle k\rangle$ to the server and move to the next round. Server:

- Collect $\mathbf{p}_{i}\langle k\rangle$ from at least $t$ parties. Let $\mathcal{U}_{3}\langle k\rangle \subseteq \mathcal{U}_{2}\langle k\rangle$ be this set of parties. Send to parties in $\mathcal{U}_{3}\langle k\rangle$ the set $\mathcal{U}_{3}\langle k\rangle$.

- Round 3 (Unmasking):

For each party $\mathcal{P}_{i}$ in parallel:

- Receive from the server a set $\mathcal{U}_{3}\langle k\rangle$. Verify that $\mathcal{U}_{3}\langle k\rangle \subseteq \mathcal{U}_{2}\langle k\rangle$, that $\left|\mathcal{U}_{3}\langle k\rangle\right| \geq t$. If any of the above operations fails, abort

- For each other party $\mathcal{P}_{j} \in \mathcal{U}_{2}\langle k\rangle \backslash\{i\}$, decrypt the ciphertext $C_{j, i}\langle k\rangle$ (abort if decryption fails), which was received in the Round 2, to get $\left(j^{\prime}, i^{\prime}, c_{j^{\prime}}\langle k\rangle, \llbracket h_{j^{\prime}}\langle k\rangle \rrbracket_{i}, \llbracket r_{j^{\prime}}\langle k\rangle \rrbracket_{i}, \llbracket b_{j^{\prime}}\langle k\rangle \rrbracket_{i}, \llbracket \mathrm{msk}_{j^{\prime}}\langle k\rangle \rrbracket_{i}\right) \leftarrow \operatorname{SE} \cdot \operatorname{Dec}\left(C_{j, i}\langle k\rangle ; \mathrm{k}_{i, j}\langle k\rangle\right)$. Assert $j^{\prime}=j$ and $i^{\prime}=i$.

- Send its secret seed for self-mask and two sets of shares to the server: $\left(b_{i}\langle k\rangle,\left\{\llbracket b_{j}\langle k\rangle \rrbracket_{i}\right\}_{j \in \mathcal{U}_{3}}\langle k\rangle,\left\{\llbracket \mathrm{msk}_{j}\langle k\rangle \rrbracket_{i}\right\}_{j \in \mathcal{U}_{2}}\langle k\rangle \backslash \mathcal{U}_{3}\langle k\rangle\right)$. Server:

- Collect message from at least $t$ parties. Let $\mathcal{U}_{4}\langle k\rangle \subseteq \mathcal{U}_{3}\langle k\rangle$ be this set of parties. If $\left|\mathcal{U}_{4}\langle k\rangle\right|<t$, abort.

- For each $\mathcal{P}_{i} \in \mathcal{U}_{3}\langle k\rangle \backslash \mathcal{U}_{4}\langle k\rangle$, reconstruct $b_{i}\langle k\rangle \leftarrow$ SS.Combine $\left(t,\left\{\llbracket b_{i}\langle k\rangle \rrbracket_{j}\right\}_{j \in \mathcal{U}_{4}\langle k\rangle}\right)$. For each $\mathcal{P}_{i} \in \mathcal{U}_{2}\langle k\rangle \backslash \mathcal{U}_{3}\langle k\rangle$, reconstruct

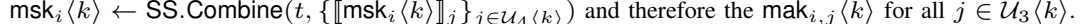

- Compute the aggregation result $\mathbf{a}\langle k\rangle \leftarrow \sum_{i \in \mathcal{U}_{3}\langle k\rangle} \mathbf{p}_{i}\langle k\rangle-\sum_{i \in \mathcal{U}_{3}\langle k\rangle} \operatorname{PRG}\left(b_{i}\langle k\rangle\right)+\sum_{i \in \mathcal{U}_{2}}\langle k\rangle \backslash \mathcal{U}_{3}\langle k\rangle, j \in \mathcal{U}_{3}\langle k\rangle \Delta_{i, j} \mathrm{PRG}\left(\mathrm{mak}_{i, j}\langle k\rangle\right)$ and send $\left(\mathbf{a}\langle k\rangle, \mathcal{U}_{4}\langle k\rangle\right)$ to all parties in $\mathcal{U}_{4}\langle k\rangle$.

Fig. 4. Aggregation Phase for Privacy-preserving Verifiable Aggregation Protocol.

achieved if an adversary who wants to forge the aggregation result after all clients having committed their homomorphic hash values respectively can be detected with an overwhelming probability.

Definition 3 (Integrity of aggregation). In the $k$-th epoch, let $\boldsymbol{v}_{H}\langle k\rangle$ be the partially aggregation result of the inputs of honest parties in $\mathcal{U}_{3}\langle k\rangle$, and $\boldsymbol{v}_{i}\langle k\rangle$ be the well-formed input of some corrupted party $\mathcal{P}_{i} \in \mathcal{U}_{3}\langle k\rangle \cap \mathcal{C}$ whose hash was computed and committed in ShareMetadata. We say that the integrity of aggregation in a verification batch of size $\ell$ holds, if an adversary can have honest parties accept its forged aggregation result in some epochs of this batch with a negligible probability, i.e.,

$$
\begin{aligned}
& \operatorname{Pr}\left[\begin{array}{l|l}
\mathcal{P}_{i} \text { outputs } \perp & \begin{array}{l}
\mathcal{P}_{i} \in \mathcal{V}_{2} \backslash \mathcal{C}, \text { for some } k \in \mathcal{K} \subseteq[\ell], \\
\boldsymbol{a}\langle k\rangle \leftarrow \boldsymbol{v}_{H}\langle k\rangle+\sum_{i \in \mathcal{U}_{3}\langle k\rangle \cap \mathcal{C}} \boldsymbol{v}_{i}\langle k\rangle, \\
\boldsymbol{a}\langle k\rangle \in \mathbb{Z}_{B}^{d}, \\
\boldsymbol{a}^{\prime}\langle k\rangle \leftarrow M_{\mathcal{C}}\left(\mathcal{S}, k, T_{k}, r_{\mathcal{C}}\right), \\
\boldsymbol{a}^{\prime}\langle k\rangle \in \mathbb{Z}_{B}^{d}, \boldsymbol{a}^{\prime}\langle k\rangle \neq \boldsymbol{a}\langle k\rangle
\end{array}
\end{array}\right] \\
& \geq 1-\operatorname{neg} /(\kappa) \text {. }
\end{aligned}
$$

By Definition 4, we define input privacy of each client in the existence of the considered adversary. This definition aims to capture an adversary who corrupts the server and a subset of parties learns nothing from protocol transcript but the partially aggregation result of honest parties. We say that an adversary learns nothing if its view can be simulated by a simulator without any secret internal state of honest parties (e.g., the knowledge of the gradient of a client). More specifically, input 
Protocol $\Pi_{\text {VerIFL }}$ (cont.)

1) Batch verification phase (of batch size $\ell$ ):

- Round 0 (Decommitting):

For each party $\mathcal{P}_{i}$ in parallel:

- Send $\left(\left\{h_{i}\langle k\rangle, r_{i}\langle k\rangle\right\}_{k \in[\ell]}\right)$ to the server.

Server:

- Collect messages from at least $t$ parties. Let $\mathcal{V}_{1} \subseteq \mathcal{U}_{4}\langle\ell\rangle$ be this set of parties (note that $\mathcal{U}_{4}\langle\ell\rangle$ is the latest set of surviving parties in this batch of size $\ell$ ). If

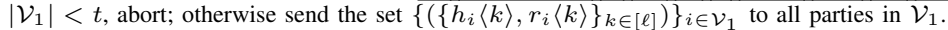

- Round 1 (DroppedDecommitting):

For each party $\mathcal{P}_{i}$ in parallel:

- Receive from the server the set $\left\{\left(\left\{h_{i}\langle k\rangle, r_{i}\langle k\rangle\right\}_{k \in[\ell]}\right)\right\}_{i \in \mathcal{V}_{1}}$ and infer the set $\mathcal{V}_{1}$. If $\left|\mathcal{V}_{1}\right|<t$, abort.

- Send two sets of shares to the server: $\left\{\llbracket h_{j}\langle k\rangle \rrbracket_{i}\right\}_{k \in[\ell], j \in \mathcal{U}_{3}\langle k\rangle \backslash \mathcal{V}_{1}}$ and $\left\{\llbracket r_{j}\langle k\rangle \rrbracket_{i}\right\}_{k \in[\ell], j \in \mathcal{U}_{3}\langle k\rangle \backslash \mathcal{V}_{1}}$ Server:

- Collect sets of shares from at least $t$ parties. Let $\mathcal{V}_{2}$ be this set of parties. If $\mathcal{V}_{2}<t$, abort.

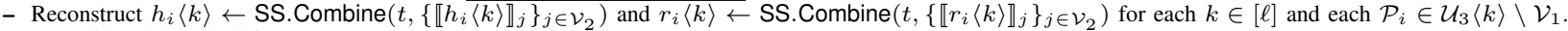

- Send $\left\{\left(h_{i}\langle k\rangle, r_{i}\langle k\rangle\right)\right\}_{k \in[\ell], i \in \mathcal{U}_{3}\langle k\rangle \backslash \mathcal{V}_{1}}$ to all parties in $\mathcal{V}_{2}$.

- Round 2 (BatchChecking):

For each party $\mathcal{P}_{i}$ in parallel:

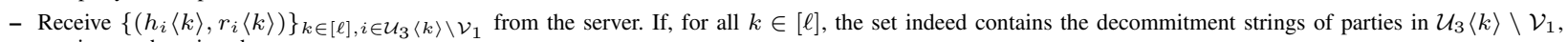
continue; otherwise abort.

- Check that COM.Decommit $\left(c_{j}\langle k\rangle, h_{j}\langle k\rangle, r_{j}\langle k\rangle\right)=1$ holds for all $k \in[\ell]$ and $j \in \mathcal{U}_{3}\langle k\rangle \backslash\{i\}$; otherwise output $\perp$.

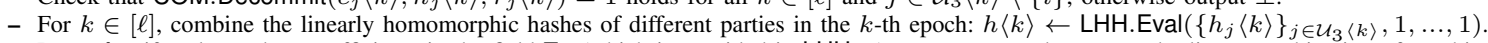

- Draw $\ell$ uniformly random coefficients in the field $\mathbb{F}_{q}$ (which is provided in LHHpp) $\alpha_{1}, \ldots, \alpha_{\ell}$ and compute the linear combination of combined hashes of

different epochs: $h^{*} \leftarrow$ LHH.Eval $\left(h\langle 1\rangle, \ldots, h\langle\ell\rangle, \alpha_{1}, \ldots, \alpha_{\ell}\right)$.

- Compute the linear combination of the aggregation results of different epochs using the same coefficients: $\mathbf{a}^{*} \leftarrow \sum_{k \in[\ell]} \alpha_{k} \mathbf{a}\langle k\rangle \bmod B$.

- Check that $h^{*}=$ LHH.Hash $\left(\mathbf{a}^{*}\right)$. Output $(\mathbf{a}\langle 1\rangle, \ldots, \mathbf{a}\langle\ell\rangle)$ if it holds; otherwise output $\perp$.

Fig. 5. Verification Phase for Privacy-preserving Verifiable Aggregation Protocol.

privacy is defined in the sense that the view of an adversary corrupting less than $t$ data parties can be simulated only given the partially aggregation result of honest parties.

Definition 4 (Input privacy). We say that the input privacy of an honest client holds, if there exists a PPT simulator SIM such that for any set of parties $\mathcal{P}$, threshold $t$, security parameter $\kappa$, batch size $\ell$, set of inputs $\left\{\boldsymbol{v}_{i}\right\}_{i \in \mathcal{P}}$, $\left\{\mathcal{U}_{1}\langle k\rangle, \mathcal{U}_{2}\langle k\rangle, \mathcal{U}_{3}\langle k\rangle, \mathcal{U}_{4}\langle k\rangle\right\}_{k \in[\ell]}$ and $\mathcal{V}_{1}, \mathcal{V}_{2}$ such that for all $j \in[3]$ and $k \in[\ell-1]$

$$
\begin{aligned}
& \mathcal{P} \supseteq \mathcal{U}_{1}\langle 1\rangle, \\
& \mathcal{U}_{j}\langle k\rangle \supseteq \mathcal{U}_{j+1}\langle k\rangle, \\
& \mathcal{U}_{4}\langle k\rangle \supseteq \mathcal{U}_{1}\langle k+1\rangle, \\
& \mathcal{U}_{4}\langle\ell\rangle \supseteq \mathcal{V}_{1} \supseteq \mathcal{V}_{2},
\end{aligned}
$$

and set of corrupted parties $\mathcal{C}$ such that $\mathcal{C} \subseteq \mathcal{P} \cap\{\mathcal{S}\}$ and $|\mathcal{C} \backslash\{\mathcal{S}\}|<t$, the output of SIM is computationally indistinguishable from the output of $R E A L_{\mathcal{C}}^{\mathcal{P}, t, \kappa}$ :

$\operatorname{REAL}_{\mathcal{C}}^{\mathcal{P}, t, \kappa}\left(\left\{\boldsymbol{v}_{i}\right\}_{i \in \mathcal{P}},\left\{\mathcal{U}_{1}\langle k\rangle, \mathcal{U}_{2}\langle k\rangle, \mathcal{U}_{3}\langle k\rangle, \mathcal{U}_{4}\langle k\rangle\right\}_{k \in[\ell]}, \mathcal{V}_{1}, \mathcal{V}_{2}\right)$

$\approx_{c}$

$\operatorname{SIM}_{\mathcal{C}}^{\mathcal{P}, t, \kappa}\left(\left\{\boldsymbol{v}_{i}\right\}_{i \in \mathcal{C}},\{z\langle k\rangle\}_{k \in[\ell]},\left\{\mathcal{U}_{1}\langle k\rangle, \mathcal{U}_{2}\langle k\rangle, \mathcal{U}_{3}\langle k\rangle, \mathcal{U}_{4}\langle k\rangle\right\}_{k \in[\ell]}, \mathcal{V}_{1}, \mathcal{V}_{2}\right)$ where

$$
\boldsymbol{z}\langle k\rangle= \begin{cases}\sum_{i \in \mathcal{U}_{3}\langle k\rangle \backslash \mathcal{C}} \boldsymbol{v}_{i}, & \text { if }\left|\mathcal{U}_{3}\langle k\rangle\right| \geq t \\ \perp, & \text { otherwise. }\end{cases}
$$

Lemma 1. Assume the hardness of discrete logarithm and the security of COM. In the AMOTIZED-VERIFICATION step, an honest client will accept the aggregation results $\boldsymbol{a}\langle 1\rangle, \ldots, \boldsymbol{a}\langle\ell\rangle$ if and only if these results are honestly aggregated by the server with an overwhelming probability.

Proof. Assume there exists a PPT adversary that succeeds in letting some honest party $\mathcal{P}_{i}$ output its forged aggregation results $\overline{\mathbf{a}}\langle k\rangle \neq \mathbf{a}\langle k\rangle=\sum_{i} \mathbf{v}_{i}\langle k\rangle$ for all $k \in \mathcal{K} \subseteq[\ell]$ in BatchChecking. We first observe that, since $\mathcal{P}_{i}$ does not output $\perp$, the decommitment in BatchChecking should be done successfully. For decommitment strings $\left(h_{j}\langle k\rangle, r_{j}\langle k\rangle\right)$ where $k \in \mathcal{K}$ and $j \in \mathcal{U}_{3}\langle k\rangle \cap \mathcal{C}$, the adversary has the freedom whether to send correct decommitment strings in Decommitting. If it chooses to send malformed decommitment strings, the decommitment in BatchChecking fails with a non-negligible probability since COM is binding. The same argument applies to the case where the adversary sends to $\mathcal{P}_{i}$ its incorrectly reconstructed decommitment strings of (honest) dropped parties in DroppedDecommitting.

The above argument implies that the adversary cannot change the hash values it has committed in ShareMetadata on behalf of corrupted parties without having $\mathcal{P}_{i}$ output $\perp$. Recall that, by protocol specification, the honestly aggregation result $\mathbf{a}\langle k\rangle$ and the final hash $h^{*}$ satisfy

$$
\begin{aligned}
h^{*} & =\operatorname{LHH} . H a s h\left(\mathbf{a}^{*}\right)=\text { LHH.Hash }\left(\sum_{k \in[\ell]} \alpha_{k} \mathbf{a}\langle k\rangle\right) \\
& =\prod_{j \in[d]} g_{j}^{\sum_{k \in[\ell]} \alpha_{k} \mathbf{a}\langle k\rangle[j]} \\
& =\left(\prod_{j \in[d]} g_{j}^{\sum_{k \in \mathcal{K}} \alpha_{k} \mathbf{a}\langle k\rangle[j]}\right) \cdot\left(\prod_{j \in[d]} g_{j}^{\sum_{k \in[\ell] \backslash \mathcal{K}} \alpha_{k} \mathbf{a}\langle k\rangle[j]}\right)
\end{aligned}
$$

Meanwhile, with unchanged hashes committed in ShareMetadata, we have another linear combination 
$\overline{\mathbf{a}} \leftarrow \sum_{k \in \mathcal{K}} \alpha_{k} \overline{\mathbf{a}}\langle k\rangle+\sum_{k \in[\ell] \backslash \mathcal{K}} \alpha_{k} \mathbf{a}\langle k\rangle$ and therefore $\mathrm{LHH} . \operatorname{Hash}(\overline{\mathbf{a}})$

$$
\begin{aligned}
& =\operatorname{LHH} . \text { Hash }\left(\sum_{k \in \mathcal{K}} \alpha_{k} \overline{\mathbf{a}}\langle k\rangle+\sum_{k \in[\ell] \backslash \mathcal{K}} \alpha_{k} \mathbf{a}\langle k\rangle\right) \\
& =\left(\prod_{j \in[d]} g_{j}^{\sum_{k \in \mathcal{K}} \alpha_{k} \overline{\mathbf{a}}\langle k\rangle[j]}\right) \cdot\left(\prod_{j \in[d]} g_{j}^{\sum_{k \in[\ell] \backslash \mathcal{K}} \alpha_{k} \mathbf{a}\langle k\rangle[j]}\right) .
\end{aligned}
$$

Since $\mathcal{P}_{i}$ does not output $\perp$, it should hold that $h^{*}=$ LHH.Hash $(\overline{\mathbf{a}})$, i.e.,

$$
\prod_{j \in[d]} g_{j}^{\sum_{k \in \mathcal{K}} \alpha_{k} \mathbf{a}\langle k\rangle[j]}=\prod_{j \in[d]} g_{j}^{\sum_{k \in \mathcal{K}} \alpha_{k} \overline{\mathbf{a}}\langle k\rangle[j]} .
$$

where $\mathbf{a}\langle k\rangle \neq \overline{\mathbf{a}}\langle k\rangle$ for $k \in \mathcal{K}$. Notice that the coefficients $\alpha_{k}$ for $k \in \mathcal{K}$ are uniformly drawn from $\mathbb{F}_{q}$, the event that $h^{*}=\mathrm{LHH} . \mathrm{Hash}(\overline{\mathbf{a}})$ and

$$
\sum_{k \in \mathcal{K}} \mathbf{a}\langle k\rangle[j]=\sum_{k \in \mathcal{K}} \overline{\mathbf{a}}\langle k\rangle[j]
$$

in which $j \in[d]$ and $\mathbf{a}\langle k\rangle \neq \overline{\mathbf{a}}\langle k\rangle$ for $k \in \mathcal{K}$ happens with a probability $1 /\left|\mathbb{F}_{q}\right|$, which is negligible in the security parameter $\kappa$. In other words, conditioned on $h^{*}=\operatorname{LHH} . \operatorname{Hash}(\overline{\mathbf{a}})$, it is hold that

$$
\sum_{k \in \mathcal{K}} \mathbf{a}\langle k\rangle[j] \neq \sum_{k \in \mathcal{K}} \overline{\mathbf{a}}\langle k\rangle[j]
$$

for some $j \in[d]$, which induces a collision of LHH.

It is readily seen that such a PPT adversary can break either the binding property of COM or the collision resistance (Definition 1) of LHH with a non-negligible probability, which is infeasible under the assumption. This completes the proof.

Theorem 1. Assume the hardness of discrete logarithm and the security of COM. VERIFL achieves integrity of aggregation according to Definition 3.

Proof. This theorem is straightforward from Lemma 1.

Theorem 2. Assume the security of SE, KA, SS and COM. VERIFL achieves input privacy according to Definition 4 in the CRS-hybrid model.

Proof. The proof is identical to that for Theorem 6.3 in [7], except that we should additionally take care of the hashes being committed in ShareMetadata. Notice that SIM did not know the real inputs of honest parties by the time it was asked to compute the hash as well as the commitment. The strategy of SIM is to hash a dummy vector (e.g., the one filled with 0 's) and commit this dummy hash value. Give that COM is hiding and the secret sharing scheme hides messages being shared, the joint view w.r.t. the aggregation phase of parties in $\mathcal{C}$ will be indistinguishable from that in $\operatorname{REAL}_{\mathcal{C}}^{\mathcal{P}, t, \kappa}$.

It remains to show that the joint view w.r.t. the verification phase of parties in $\mathcal{C}$ can also be simulated by SIM. Note that the simulated commitment is committed to the dummy hash which is different (with an overwhelming probability) to that of the vector sampled by SIM after it obtains the partially aggregation result of honest parties by querying $\mathbf{z}\langle k\rangle$ for $k \in[\ell]$. Recall that COM is equivocal and, in the CRS-hybrid model, SIM has the trapdoor td which is associated with the COMpp output by COM.Setup. So, using the trapdoor td, SIM can equivocate these commitments to the hashes of inputs sampled by it on behalf of honest parties. The equivocality of COM (Definition 2) guarantees that these commitments are consistent with hashes of the simulated inputs of honest parties and therefore can be successfully decommitted with an overwhelming probability. Moreover, since $\mathcal{C}$ forms an unqualified set to reconstruct shared secrets by assumption, SIM can adjust its shares held on behalf of honest parties such that the reconstructed decomittment strings match those obtained from equivocation. That is, the verification phase can be simulated, which completes the proof.

\section{Evaluation}

\section{A. Experimental Setup}

We show the performance of our protocol based on a prototype implementation. The prototype is written in Java while we use JNI to implement low-level cryptography algorithms. In particular, linearly homomorphic hash LHH is realized using elliptic curve over the NIST P-256 curve. For efficient equivocal commitment COM, we use folklore hash commitment scheme instantiated with SHA-256, which is proved secure in restricted programmable and observable global random oracle model [30]. For secret sharing scheme, we adopt standard $t$ out-of- $N$ Shamir secret sharing. For key agreement, we use elliptic curve Diffie-Hellman over the NIST P-256 curve with SHA-384. The symmetric encryption is instantiated with AESOFB mode with 256-bits key and 128-bits IV. For pseudorandom generator, we use AES-CTR mode. In addition, we fix two moduli $R=2^{24}$ and $B=2^{34}$ (i.e., the maximum number of clients is $B / R=2^{10}=1024$ ) and assume all clients will input honestly as in our adversarial model.

We simulate clients and the aggregation server on a 64 bits Ubuntu 16.04 LTS desktop equipped with Intel i7-7700 CPU $(3.60 \mathrm{GHz})$ and $16 \mathrm{~GB}$ RAM. The simulation is singlethreaded. Given that end-to-end networking time will not significantly influence the asymptotic computation complexity of our protocol and can be calculated using bandwidth, we omit this part in our evaluation.

Dropout cases. For client dropout, we consider the following two cases of dropout in our protocol:

- Case I dropout: In this case, clients drop out of the protocol after sending their metadata to other clients via server in ShareMetadata but before sending their masked gradients to the server in MaskedlnputCollection. As discussed in [7], this dropout case will lead to the worst performance of aggregation phase in our protocol for the expensive computation overhead to recover the pairwise-mask.

- Case II dropout: In this case, some clients drop out before reporting their decommitment strings to other clients via the aggregation server in Decommitting. The server has to recover these strings of dropped clients by asking all surviving clients to send their shares of these strings and running secret reconstruction algorithm to recover 


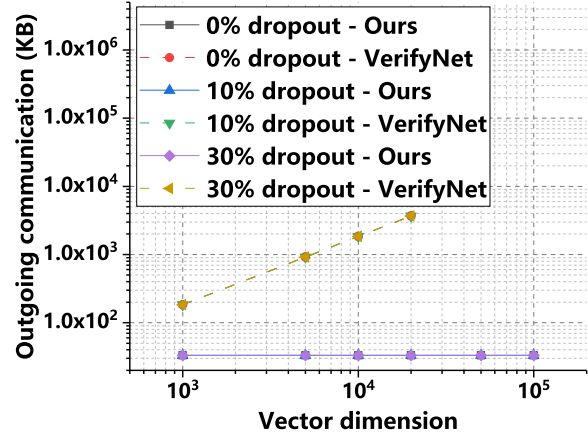

(a)

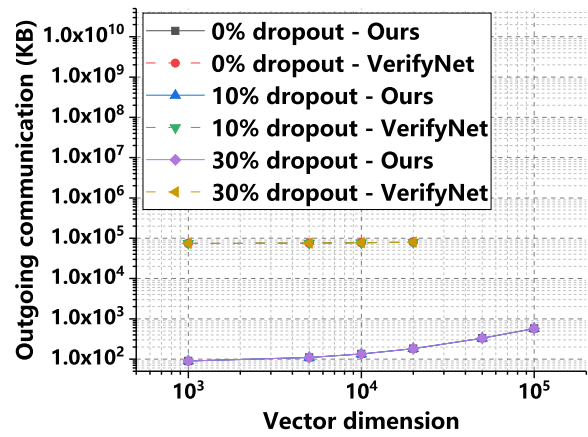

(d)

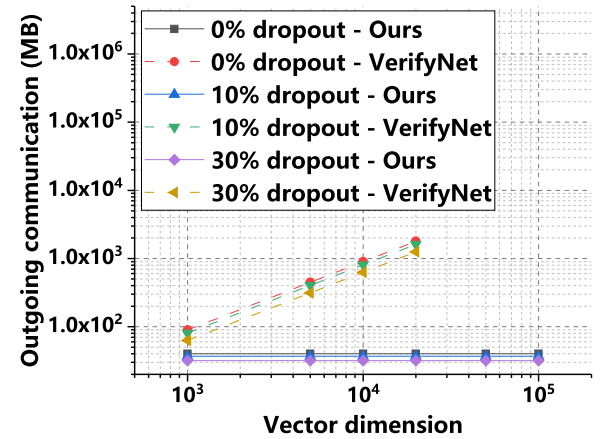

(b)

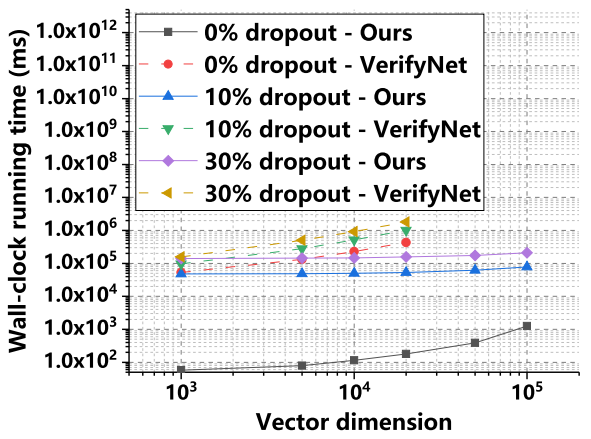

(e)

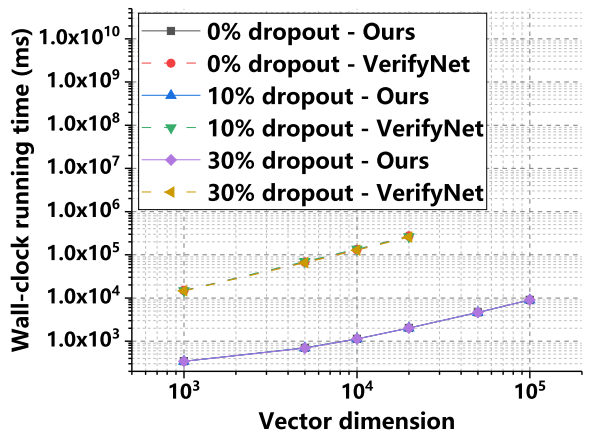

(c)

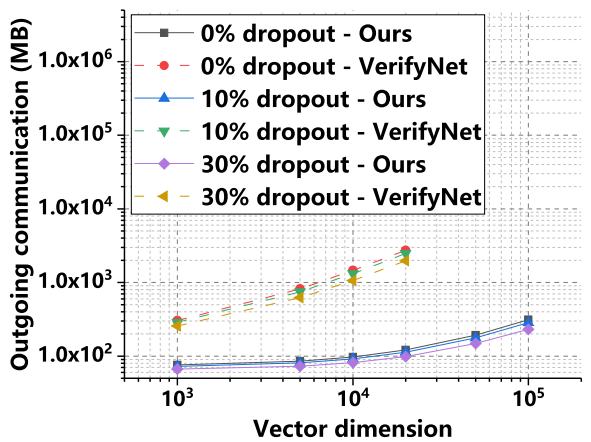

(f)

Fig. 6. Comparison between our protocol and VerifyNet [8] in terms of (i) outgoing communication overhead for verification, (ii) the total computation overhead, and (iii) the total outgoing communication overhead, as the dimension of gradient vectors increases. (a) Outgoing communication overhead for verification per client. (b) Outgoing communication overhead for verification of the server. (c) Computation overhead per client. (d) Outgoing communication overhead per client. (e) Computation overhead of the server. (f) Total outgoing communication overhead of the server.

TABLE I

COMPUTATION OVERHEAD WITH RESPECT TO CASE I DROPOUT ${ }^{1}$

\begin{tabular}{|c|c|c|c|c|c|c|c|c|c|c|c|}
\hline \multirow{2}{*}{ Num. Client } & \multirow{2}{*}{ Dropout } & & \multicolumn{5}{|c|}{ Aggregation phase (Round \#) } & \multicolumn{3}{|c|}{ Verification phase (Round \#) } & \multirow{2}{*}{ Total } \\
\hline & & & 0 & 1 & 2 & 3 & Receive result & 0 & 1 & 2 & \\
\hline \multirow{6}{*}{1000} & \multirow{2}{*}{$0.00 \%$} & Client & $0 \mathrm{~ms}$ & $391+\underline{\mathbf{3 9 4 7} \mathrm{ms}}$ & $1798 \mathrm{~ms}$ & $0 \mathrm{~ms}$ & $45 \mathrm{~ms}$ & $\underline{\mathbf{0}} \mathrm{ms}$ & $17 \mathrm{~ms}$ & $\underline{3931} \mathrm{~ms}$ & $10129 \mathrm{~ms}$ \\
\hline & & Server & $0 \mathrm{~ms}$ & $0 \overline{\mathrm{ms}}$ & $1007 \mathrm{~ms}$ & $1363 \mathrm{~ms}$ & - & $\underline{\mathbf{1}} \mathrm{ms}$ & $\overline{\mathbf{0}} \mathrm{ms}$ & $\overline{-}$ & $2371 \mathrm{~ms}$ \\
\hline & \multirow{2}{*}{$10.00 \%$} & Client & $0 \mathrm{~ms}$ & $411+\underline{4089} \mathrm{~ms}$ & $1834 \mathrm{~ms}$ & $0 \mathrm{~ms}$ & $45 \mathrm{~ms}$ & $\underline{\mathbf{0}} \mathrm{ms}$ & $15 \mathrm{~ms}$ & $\underline{3910} \mathrm{~ms}$ & $10304 \mathrm{~ms}$ \\
\hline & & Server & $0 \mathrm{~ms}$ & $0 \overline{\mathrm{ms}}$ & $930 \mathrm{~ms}$ & $216205 \mathrm{~ms}$ & - & $\underline{\overline{\mathbf{0}}} \mathrm{ms}$ & $\overline{\mathbf{0}} \mathrm{ms}$ & $\overline{-}$ & $217135 \mathrm{~ms}$ \\
\hline & \multirow{2}{*}{$30.00 \%$} & Client & $0 \mathrm{~ms}$ & $408+\underline{\mathbf{3 9 3 0}} \mathrm{ms}$ & $1791 \mathrm{~ms}$ & $0 \mathrm{~ms}$ & $45 \mathrm{~ms}$ & $\underline{\mathbf{0}} \mathrm{ms}$ & $12 \mathrm{~ms}$ & $\underline{3915} \mathrm{~ms}$ & $10101 \mathrm{~ms}$ \\
\hline & & Server & $1 \mathrm{~ms}$ & $0 \overline{\mathrm{ms}}$ & $703 \mathrm{~ms}$ & $561420 \mathrm{~ms}$ & - & $\underline{\overline{0}} \mathrm{~ms}$ & $\overline{\mathbf{0}} \mathrm{ms}$ & $\overline{-}$ & $562124 \mathrm{~ms}$ \\
\hline \multirow{6}{*}{500} & \multirow{2}{*}{$0.00 \%$} & Client & $0 \mathrm{~ms}$ & $128+\underline{\mathbf{3 9 1 9} \mathrm{ms}}$ & $896 \mathrm{~ms}$ & $0 \mathrm{~ms}$ & $44 \mathrm{~ms}$ & $\underline{\mathbf{0}} \mathrm{ms}$ & $\underline{\mathbf{8}} \mathrm{ms}$ & $3904 \mathrm{~ms}$ & $8899 \mathrm{~ms}$ \\
\hline & & Server & $0 \mathrm{~ms}$ & $0 \overline{\mathrm{ms}}$ & $501 \mathrm{~ms}$ & $679 \mathrm{~ms}$ & - & $\underline{\overline{\mathbf{0}}} \mathrm{ms}$ & $\overline{\mathbf{0}} \mathrm{ms}$ & $\overline{-}$ & $1180 \mathrm{~ms}$ \\
\hline & \multirow{2}{*}{$10.00 \%$} & Client & $0 \mathrm{~ms}$ & $123+\underline{\mathbf{3 9 5 4} \mathrm{ms}}$ & $909 \mathrm{~ms}$ & $0 \mathrm{~ms}$ & $45 \mathrm{~ms}$ & $\underline{\mathbf{0}} \mathrm{ms}$ & $\underline{7} \mathrm{~ms}$ & $3969 \mathrm{~ms}$ & $9007 \mathrm{~ms}$ \\
\hline & & Server & $1 \mathrm{~ms}$ & $0 \overline{\mathrm{ms}}$ & $466 \mathrm{~ms}$ & $43921 \mathrm{~ms}$ & - & $\underline{\overline{\mathbf{0}}} \mathrm{ms}$ & $\overline{\mathbf{0}} \mathrm{ms}$ & $\overline{-}$ & $44388 \mathrm{~ms}$ \\
\hline & \multirow{2}{*}{$30.00 \%$} & Client & $0 \mathrm{~ms}$ & $122+\underline{\mathbf{3 9 4 3}} \mathrm{ms}$ & $896 \mathrm{~ms}$ & $0 \mathrm{~ms}$ & $44 \mathrm{~ms}$ & $\underline{\mathbf{0}} \mathrm{ms}$ & $\underline{6} \mathrm{~ms}$ & $3906 \mathrm{~ms}$ & $8917 \mathrm{~ms}$ \\
\hline & & Server & $0 \mathrm{~ms}$ & $0 \overline{\mathrm{ms}}$ & $351 \mathrm{~ms}$ & $107428 \mathrm{~ms}$ & - & $\overline{\mathbf{0}} \mathrm{ms}$ & $\underline{\overline{\mathbf{0}}} \mathrm{ms}$ & $\overline{-}$ & $107779 \mathrm{~ms}$ \\
\hline
\end{tabular}

${ }^{1}$ The dimension of gradient vectors is set to $100 \mathrm{~K}$ and the batch size is 1 . The underlined bold figures stand for the costs for verification in our protocol, compared with our implementation of [7] in the same experimental environment and parameter settings.

them. This will lead to the most expensive computation overhead in our verification phase.

\section{B. Comparison with VerifyNet [8]: Dimension-Independence}

Given that the protocol in [8] does not allow Case II dropout and amortization, we consider only the Case I dropout and set the batch size to 1 in our comparison. In addition, we set the number of clients $N=500$ and fix the threshold $t=\frac{1}{2} N$. All other parameters remain unchanged as above. It is readily seen from Figure 6(a) and Figure 6(b) that, in our protocol, the outgoing communication cost for verification of either each client or the server is independent of the dimension of gradients. However, in [8], the two metrics is linearly dependent on the dimension of gradients, which results in impractical performance when the dimension is large enough. A more comprehensive comparison with respect to the overall overhead is presented in Figure 6. It is easy to see that our protocol outperforms VerifyNet completely. A more encouraging result is that our protocol can be scaled to support high-dimensional gradient vectors (e.g., 100K) with even better performance than that achieved by VerifyNet in 
TABLE II

Outgoing COMMUNiCATION OvERHEAD WITH RESPECT TO CASE I DROPOUT ${ }^{1}$

\begin{tabular}{|c|c|c|c|c|c|c|c|c|c|c|c|}
\hline \multirow{2}{*}{ Num. Client } & \multirow{2}{*}{ Dropout } & & \multicolumn{5}{|c|}{ Aggregation phase (Round \#) } & \multicolumn{3}{|c|}{ Verification phase (Round \#) } & \multirow{2}{*}{ Total } \\
\hline & & & 0 & 1 & 2 & 3 & Receive result & 0 & 1 & 2 & \\
\hline \multirow{6}{*}{1000} & \multirow{2}{*}{$0.00 \%$} & Client & $0.07 \mathrm{~KB}$ & $68.29+\mathbf{6 6 . 3 7} \mathrm{KB}$ & $488.28 \mathrm{~KB}$ & $35.16 \mathrm{~KB}$ & - & $\underline{0.07} \mathrm{~KB}$ & $\underline{0.00} \mathrm{~KB}$ & - & $658.24 \mathrm{~KB}$ \\
\hline & & Server & $66.41 \mathrm{~KB}$ & $68.29+\underline{\mathbf{9 7 . 5 6}} \mathrm{KB}$ & $1.95 \mathrm{~KB}$ & $488.28 \mathrm{~KB}$ & - & $\underline{\mathbf{6 6 . 4 1}} \mathrm{KB}$ & $\overline{\mathbf{0 . 0 0}} \mathrm{KB}$ & - & $788.90 \mathrm{~KB}$ \\
\hline & \multirow{2}{*}{$10.00 \%$} & Client & $0.07 \mathrm{~KB}$ & $68.29+\underline{\mathbf{6 6 . 3 7}} \mathrm{KB}$ & $488.28 \mathrm{~KB}$ & $35.16 \mathrm{~KB}$ & - & $\underline{0.07} \mathrm{~KB}$ & $\underline{0.00} \mathrm{~KB}$ & - & $658.24 \mathrm{~KB}$ \\
\hline & & Server & $66.41 \mathrm{~KB}$ & $68.29+\underline{\underline{\mathbf{9 7 . 5 6}}} \mathrm{KB}$ & $1.76 \mathrm{~KB}$ & $488.28 \mathrm{~KB}$ & - & $\underline{59.77} \mathrm{~KB}$ & $\underline{\overline{0.00}} \mathrm{~KB}$ & - & $782.06 \mathrm{~KB}$ \\
\hline & \multirow{2}{*}{$30.00 \%$} & Client & $0.07 \mathrm{~KB}$ & $68.29+\underline{\mathbf{6 6 . 3 7}} \mathrm{KB}$ & $488.28 \mathrm{~KB}$ & $35.16 \mathrm{~KB}$ & - & $0.07 \mathrm{~KB}$ & $\underline{0.00} \mathrm{~KB}$ & - & $658.24 \mathrm{~KB}$ \\
\hline & & Server & $66.41 \mathrm{~KB}$ & $68.29+\overline{\underline{\mathbf{9 7 . 5 6}}} \mathrm{KB}$ & $1.37 \mathrm{~KB}$ & $488.28 \mathrm{~KB}$ & - & $\overline{46.48} \mathrm{~KB}$ & $\overline{\mathbf{0 . 0 0}} \mathrm{KB}$ & - & $768.39 \mathrm{~KB}$ \\
\hline \multirow{6}{*}{500} & \multirow{2}{*}{$0.00 \%$} & Client & $0.07 \mathrm{~KB}$ & $34.11+\underline{\mathbf{3 3 . 1 7}} \mathrm{KB}$ & $488.28 \mathrm{~KB}$ & $17.58 \mathrm{~KB}$ & - & $\underline{0.07} \mathrm{~KB}$ & $\underline{0.00} \mathrm{~KB}$ & - & $573.28 \mathrm{~KB}$ \\
\hline & & Server & $33.20 \mathrm{~KB}$ & $34.11+\underline{\mathbf{4 8 . 7 3}} \mathrm{KB}$ & $0.98 \mathrm{~KB}$ & $488.28 \mathrm{~KB}$ & - & $\underline{33.20} \mathrm{~KB}$ & $\underline{\mathbf{0 . 0 0}} \mathrm{KB}$ & - & $638.51 \mathrm{~KB}$ \\
\hline & \multirow{2}{*}{$10.00 \%$} & Client & $0.07 \mathrm{~KB}$ & $34.11+\underline{\mathbf{3 3 . 1 7}} \mathrm{KB}$ & $488.28 \mathrm{~KB}$ & $17.58 \mathrm{~KB}$ & - & 0.07 KB & $\underline{0.00} \mathrm{~KB}$ & - & $573.28 \mathrm{~KB}$ \\
\hline & & Server & $33.20 \mathrm{~KB}$ & $34.11+\overline{\underline{\mathbf{4 8 . 7 3}}} \mathrm{KB}$ & $0.88 \mathrm{~KB}$ & $488.28 \mathrm{~KB}$ & - & $\overline{29.88} \mathrm{~KB}$ & $\overline{\underline{\mathbf{0 . 0 0}}} \mathrm{KB}$ & - & $635.09 \mathrm{~KB}$ \\
\hline & \multirow{2}{*}{$30.00 \%$} & Client & $0.07 \mathrm{~KB}$ & $34.11+\underline{\mathbf{3 3 . 1 7}} \mathrm{KB}$ & $488.28 \mathrm{~KB}$ & $17.58 \mathrm{~KB}$ & - & $\underline{\mathbf{0 . 0 7}} \mathrm{KB}$ & $\underline{0.00} \mathrm{~KB}$ & - & $573.28 \mathrm{~KB}$ \\
\hline & & Server & $33.20 \mathrm{~KB}$ & $34.11+\underline{\underline{48.73}} \mathrm{~KB}$ & $0.68 \mathrm{~KB}$ & $488.28 \mathrm{~KB}$ & - & $\underline{\underline{23.24}} \mathrm{~KB}$ & $\overline{\underline{\mathbf{0 . 0 0}}} \mathrm{KB}$ & - & $628.25 \mathrm{~KB}$ \\
\hline
\end{tabular}

${ }^{1}$ The dimension of gradient vectors is set to $100 \mathrm{~K}$ and the batch size is 1 . The entries of server are outgoing communication sent from the server to a single client. The underlined bold figures stand for the costs for verification in our protocol, compared with our implementation of [7] in the same experimental environment and parameter settings.

TABLE III

COMPUTATION OVERHEAD WITH RESPECT TO CASE II DROPOUT ${ }^{1}$

\begin{tabular}{|c|c|c|c|c|c|c|c|c|c|c|c|}
\hline \multirow{2}{*}{ Num. Client } & \multirow{2}{*}{ Dropout } & & \multicolumn{5}{|c|}{ Aggregation phase (Round \#) } & \multicolumn{3}{|c|}{ Verification phase (Round \#) } & \multirow{2}{*}{ Total } \\
\hline & & & 0 & 1 & 2 & 3 & Receive result & 0 & 1 & 2 & \\
\hline \multirow{6}{*}{1000} & \multirow{2}{*}{$0.00 \%$} & Client & $0 \mathrm{~ms}$ & $392+\underline{\mathbf{3 9 4 6}} \mathrm{ms}$ & $1798 \mathrm{~ms}$ & $0 \mathrm{~ms}$ & $45 \mathrm{~ms}$ & $\underline{0} \mathrm{~ms}$ & $17 \mathrm{~ms}$ & $3931 \mathrm{~ms}$ & $10129 \mathrm{~ms}$ \\
\hline & & Server & $0 \mathrm{~ms}$ & $0 \overline{\mathrm{ms}}$ & $1007 \mathrm{~ms}$ & $1363 \mathrm{~ms}$ & - & $\underline{\overline{1}} \mathrm{~ms}$ & $\overline{\mathbf{0}} \mathrm{ms}$ & $\overline{-}$ & $2371 \mathrm{~ms}$ \\
\hline & \multirow{2}{*}{$10.00 \%$} & Client & $0 \mathrm{~ms}$ & $405+\underline{\mathbf{3 9 3 1} \mathrm{ms}}$ & $1792 \mathrm{~ms}$ & $0 \mathrm{~ms}$ & $45 \mathrm{~ms}$ & $\underline{\mathbf{0}} \mathrm{ms}$ & $\underline{15} \mathrm{~ms}$ & $3927 \mathrm{~ms}$ & $10115 \mathrm{~ms}$ \\
\hline & & Server & $0 \mathrm{~ms}$ & $0 \overline{\mathrm{ms}}$ & $1004 \mathrm{~ms}$ & $1358 \mathrm{~ms}$ & - & $\overline{\mathbf{0}} \mathrm{ms}$ & $\underline{17 \overline{2309}} \mathrm{~ms}$ & $\overline{-}$ & $174671 \mathrm{~ms}$ \\
\hline & \multirow{2}{*}{$30.00 \%$} & Client & $0 \mathrm{~ms}$ & $408+\underline{\mathbf{3 9 3 0}} \mathrm{ms}$ & $1796 \mathrm{~ms}$ & $0 \mathrm{~ms}$ & $45 \mathrm{~ms}$ & $\underline{\mathbf{0}} \mathrm{ms}$ & $\underline{12} \mathrm{~ms}$ & $3925 \mathrm{~ms}$ & $10116 \mathrm{~ms}$ \\
\hline & & Server & $0 \mathrm{~ms}$ & $0 \overline{\mathrm{ms}}$ & $1011 \mathrm{~ms}$ & $1369 \mathrm{~ms}$ & - & $\underline{\overline{0}} \mathrm{~ms}$ & $\underline{51 \overline{7306}} \mathrm{~ms}$ & $\overline{-}$ & $519686 \mathrm{~ms}$ \\
\hline \multirow{6}{*}{500} & \multirow{2}{*}{$0.00 \%$} & Client & $0 \mathrm{~ms}$ & $126+\underline{\mathbf{3 9 2 1} \mathrm{ms}}$ & $896 \mathrm{~ms}$ & $0 \mathrm{~ms}$ & $44 \mathrm{~ms}$ & $0 \mathrm{~ms}$ & $8 \mathrm{~ms}$ & $3904 \mathrm{~ms}$ & $8899 \mathrm{~ms}$ \\
\hline & & Server & $0 \mathrm{~ms}$ & $0 \overline{\mathrm{ms}}$ & $501 \mathrm{~ms}$ & $679 \mathrm{~ms}$ & - & $\overline{\mathbf{0}} \mathrm{ms}$ & $\overline{\mathbf{0}} \mathrm{ms}$ & $\overline{-}$ & $1180 \mathrm{~ms}$ \\
\hline & \multirow{2}{*}{$10.00 \%$} & Client & $0 \mathrm{~ms}$ & $140+3917 \mathrm{~ms}$ & $896 \mathrm{~ms}$ & $0 \mathrm{~ms}$ & $45 \mathrm{~ms}$ & $0 \mathrm{~ms}$ & $7 \mathrm{~ms}$ & $3912 \mathrm{~ms}$ & $8917 \mathrm{~ms}$ \\
\hline & & Server & $0 \mathrm{~ms}$ & $0 \overline{\mathrm{ms}}$ & $502 \mathrm{~ms}$ & $680 \mathrm{~ms}$ & - & $\overline{\overline{0}} \mathrm{~ms}$ & $\underline{\mathbf{2 1 3}} \overline{\mathbf{3} 77} \mathrm{~ms}$ & $\overline{-}$ & $22559 \mathrm{~ms}$ \\
\hline & \multirow{2}{*}{$30.00 \%$} & Client & $0 \mathrm{~ms}$ & $136+\underline{\mathbf{3 9 2 8}} \mathrm{ms}$ & $899 \mathrm{~ms}$ & $0 \mathrm{~ms}$ & $45 \mathrm{~ms}$ & $\underline{\mathbf{0}} \mathrm{ms}$ & $\underline{6} \mathrm{~ms}$ & $\underline{3938} \mathrm{~ms}$ & $8952 \mathrm{~ms}$ \\
\hline & & Server & $1 \mathrm{~ms}$ & $0 \mathrm{~ms}$ & $507 \mathrm{~ms}$ & $681 \mathrm{~ms}$ & - & $\overline{\mathbf{0}} \mathrm{ms}$ & $64 \overline{4} 62 \mathrm{~ms}$ & - & $65651 \mathrm{~ms}$ \\
\hline
\end{tabular}

${ }^{1}$ The dimension of gradient vectors is set to $100 \mathrm{~K}$ and the batch size is 1 . The underlined bold figures stand for the costs for verification in our protocol, compared with our implementation of [7] in the same experimental environment and parameter settings.

TABLE IV

Outgoing COMMUnicATION OVERHEAD With RESPECT to CASE II DRopout ${ }^{1}$.

\begin{tabular}{|c|c|c|c|c|c|c|c|c|c|c|c|}
\hline \multirow{2}{*}{ Num. Client } & \multirow{2}{*}{ Dropout } & & \multicolumn{5}{|c|}{ Aggregation phase (Round \#) } & \multicolumn{3}{|c|}{ Verification phase (Round \#) } & \multirow{2}{*}{ Total } \\
\hline & & & 0 & 1 & 2 & 3 & Receive result & 0 & 1 & 2 & \\
\hline \multirow{3}{*}{1000} & $0.00 \%$ & $\begin{array}{l}\text { Client } \\
\text { Server }\end{array}$ & $\begin{array}{c}0.07 \mathrm{~KB} \\
66.41 \mathrm{~KB}\end{array}$ & $\begin{array}{l}68.29+\underline{\mathbf{6 6 . 3 7}} \mathrm{KB} \\
68.29+\underline{\mathbf{9 7 . 5 6}} \mathrm{KB}\end{array}$ & $\begin{array}{c}488.28 \mathrm{~KB} \\
1.95 \mathrm{~KB}\end{array}$ & $\begin{array}{c}35.16 \mathrm{~KB} \\
488.28 \mathrm{~KB}\end{array}$ & $\begin{array}{l}- \\
-\end{array}$ & $\begin{array}{r}\underline{\mathbf{0 . 0 7}} \mathrm{KB} \\
\underline{\mathbf{6 6 . 4 1}} \mathrm{KB}\end{array}$ & $\underline{\underline{0.00}} \mathrm{~KB}$ & - & $\begin{array}{l}658.24 \mathrm{~KB} \\
788.90 \mathrm{~KB}\end{array}$ \\
\hline & $10.00 \%$ & $\begin{array}{l}\text { Client } \\
\text { Server }\end{array}$ & $\begin{array}{c}0.07 \mathrm{~KB} \\
66.41 \mathrm{~KB}\end{array}$ & $\begin{array}{l}68.29+\underline{\mathbf{6 6 . 3 7}} \mathrm{KB} \\
68.29+\underline{\mathbf{9 7 . 5 6}} \mathrm{KB}\end{array}$ & $\begin{array}{c}488.28 \mathrm{~KB} \\
1.95 \mathrm{~KB}\end{array}$ & $\begin{array}{c}35.16 \mathrm{~KB} \\
488.28 \mathrm{~KB}\end{array}$ & - & $\begin{array}{r}\mathbf{0 . 0 7} \mathrm{KB} \\
\mathbf{5 9 . 7 7} \mathrm{KB} \\
\end{array}$ & 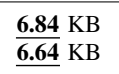 & - & $\begin{array}{l}665.07 \mathrm{~KB} \\
788.90 \mathrm{~KB}\end{array}$ \\
\hline & $30.00 \%$ & $\begin{array}{l}\text { Client } \\
\text { Server }\end{array}$ & $\begin{array}{c}0.07 \mathrm{~KB} \\
66.41 \mathrm{~KB}\end{array}$ & $\begin{array}{l}68.29+\underline{\mathbf{6 6 . 3 7}} \mathrm{KB} \\
68.29+\underline{\mathbf{9 7 . 5 6}} \mathrm{KB}\end{array}$ & $\begin{array}{c}488.28 \mathrm{~KB} \\
1.95 \mathrm{~KB}\end{array}$ & $\begin{array}{c}35.16 \mathrm{~KB} \\
488.28 \mathrm{~KB}\end{array}$ & $\begin{array}{l}- \\
-\end{array}$ & $\begin{array}{r}\underline{0.07} \mathrm{~KB} \\
\underline{\mathbf{4 6 . 4 8}} \mathrm{KB}\end{array}$ & $\begin{array}{l}\text { 20.51 KB } \\
\underline{19.92} \text { KB }\end{array}$ & $\begin{array}{l}- \\
-\end{array}$ & $\begin{array}{l}678.75 \mathrm{~KB} \\
788.90 \mathrm{~KB}\end{array}$ \\
\hline \multirow{3}{*}{500} & $0.00 \%$ & $\begin{array}{l}\text { Client } \\
\text { Server }\end{array}$ & $\begin{array}{c}0.07 \mathrm{~KB} \\
33.20 \mathrm{~KB} \\
\end{array}$ & $\begin{array}{l}34.11+\underline{\mathbf{3 3 . 1 7}} \mathrm{KB} \\
34.11+\underline{\mathbf{4 8 . 7 3}} \mathrm{KB} \\
\end{array}$ & $\begin{array}{c}488.28 \mathrm{~KB} \\
0.98 \mathrm{~KB} \\
\end{array}$ & $\begin{array}{r}17.58 \mathrm{~KB} \\
488.28 \mathrm{~KB}\end{array}$ & $\begin{array}{l}- \\
-\end{array}$ & $\begin{array}{r}\mathbf{0 . 0 7} \mathrm{KB} \\
\underline{\mathbf{3 3 . 2 0}} \mathrm{KB} \\
\end{array}$ & $\underline{\underline{0.00}} \mathrm{~KB}$ & $\begin{array}{l}- \\
-\end{array}$ & $\begin{array}{l}573.28 \mathrm{~KB} \\
638.51 \mathrm{~KB}\end{array}$ \\
\hline & $10.00 \%$ & $\begin{array}{l}\text { Client } \\
\text { Server }\end{array}$ & $\begin{array}{r}0.07 \mathrm{~KB} \\
33.20 \mathrm{~KB}\end{array}$ & $\begin{array}{l}34.11+\mathbf{3 3 . 1 7} \mathrm{KB} \\
34.11+\underline{\mathbf{4 8 . 7 3}} \mathrm{KB}\end{array}$ & $\begin{array}{c}488.28 \mathrm{~KB} \\
0.98 \mathrm{~KB}\end{array}$ & $\begin{array}{r}17.58 \mathrm{~KB} \\
488.28 \mathrm{~KB}\end{array}$ & - & $\begin{array}{r}\mathbf{0 . 0 7} \text { KB } \\
\underline{29.88} \text { KB } \\
\end{array}$ & $\begin{array}{l}\mathbf{3 . 4 2} \mathrm{KB} \\
\mathbf{3 . 3 2} \\
\mathrm{KB}\end{array}$ & - & $\begin{array}{l}576.70 \mathrm{~KB} \\
638.51 \mathrm{~KB}\end{array}$ \\
\hline & $30.00 \%$ & $\begin{array}{l}\text { Client } \\
\text { Server }\end{array}$ & $\begin{array}{c}0.07 \mathrm{~KB} \\
33.20 \mathrm{~KB}\end{array}$ & $\begin{array}{l}34.11+\mathbf{3 3 . 1 7} \mathrm{KB} \\
34.11+\underline{\mathbf{4 8 . 7 3}} \mathrm{KB}\end{array}$ & $\begin{array}{c}488.28 \mathrm{~KB} \\
0.98 \mathrm{~KB}\end{array}$ & $\begin{array}{c}17.58 \mathrm{~KB} \\
488.28 \mathrm{~KB}\end{array}$ & - & $\begin{array}{l}\underline{0.07} \mathrm{~KB} \\
\underline{\mathbf{2 3 . 2 4}} \mathrm{KB}\end{array}$ & $\begin{array}{l}\mathbf{1 0 . 2 6} \mathrm{KB} \\
\underline{9.96} \mathrm{~KB}\end{array}$ & $\begin{array}{l}- \\
-\end{array}$ & $\begin{array}{l}583.53 \mathrm{~KB} \\
638.51 \mathrm{~KB}\end{array}$ \\
\hline
\end{tabular}

${ }^{1}$ The dimension of gradient vectors is set to $100 \mathrm{~K}$ and the batch size is 1 . The entries of server are outgoing communication sent from the server to a single client. The underlined bold figures stand for the costs for verification in our protocol, compared with our implementation of [7] in the same experimental environment and parameter settings.

dealing with low-dimensional one.

C. Comparison with Secure Aggregation [7]

In Table I, Table II, Table III and Table IV, we give out the additional costs for verification in our protocol. The baseline 


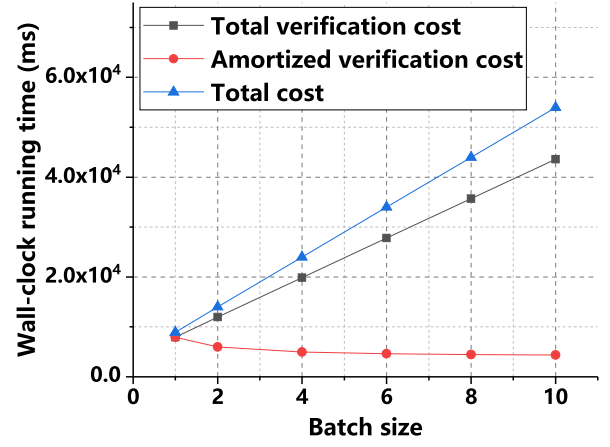

Fig. 7. Comparison between total computation cost for verification, amortized computation cost for verification and total computation cost per client. The dimension of gradient vectors is set to $100 \mathrm{~K}$ and the number of clients is 500 . Assume no dropout.

protocol is [7] that addresses the input privacy of mobile clients. It is easy to see from underlined bold figures that our protocol does not introduce too much additional communication overhead to the baseline secure aggregation protocol per client. For example, when the dimension of gradient vectors is set to $100 \mathrm{~K}$, the communication for transferring a gradient is always $488.28 \mathrm{~KB}$, which takes the largest proportion of the overall communication per client. Compared with that, the additional communication caused by verification protocol is insignificant. As for the computation overhead, the one caused by our protocol is also affordable compared with the one in [7]. In the real-world applications of FL, it is not uncommon that each local iteration of machine learning requires tens of seconds. When deploying our protocol to these applications where dropout is not severe (e.g., $<1 \%$ occurred naturally as reported in [7]), the overall computation overhead will not blow up since our protocol has similar wall-clock running time with a local iteration of machine learning.

\section{Other Experimental Results}

Amortized Verification. In Figure 7, it is clear that, with the growth of batch size, the amortized verification overhead per epoch can be reduced nearly to that of a single LHH.Hash call. In other words, with a large enough batch size, we can approximately halve the computation overhead of a client in the sense of amortization. However, in real-world applications where there are dropouts in each epoch, the batch size cannot be set too large given that too large verification batch will lead to severe accumulation of dropouts and therefore the expensive overhead to run secret reconstruction algorithm. We regard this phenomenon as a tradeoff and therefore the batch size should be fine-tuned in real-world applications.

Number of clients. Both the computation and communication cost in client side grows linearly in the number of clients, which results from the fact that the number of secret shares generated in ShareMetadata is proportional to the number of clients. For the wall-clock running time of the server, it grows quadratically in the number of clients. The reason is that, in Unmasking, the server has to run secret reconstruction algorithm to recover the secret mask key of dropped clients and both the threshold $t$ and the number of dropped clients are proportional to the number of clients $N$.

Dropout. As shown in Figure 8 and Figure 9, it is clear that, in client side, either the computation cost or the communication cost is independent of how many other clients drop out of the protocol execution. However, the two metrics in server side is influenced by the dropout rate, especially the wall-clock running time of the server. We note that the overhead suffered by the server is acceptable.

\section{RELATED WORK}

In this section, we briefly discuss the works related to secure verifiable aggregation in federated learning.

Generic maliciously-secure multiparty computation. In general, FL involves an aggregation server and a set of clients and therefore can be regarded as a specific multiparty computation problem. Although there are piles of works [14], [15], [16], [17], [18], [19], [20], [21], [31] guarantee the integrity of computation in the existence of a malicious (i.e., active) adversary, they are not suitable for FL settings. For garbled circuit-based protocols [19], [20], [21], [31], they deal with the malicious adversary at the cost of expensive communication overhead (e.g., that led by commit-and-prove [18] or cut-andchoose [32], [21] technique) and cannot be deployed on a large scale. For secret sharing-based protocols [14], [15], [16], each client has to divide each entry of its input into (additive) shares and send them to each other client, which results in a rather expensive communication overhead in $O(N d)$. In addition, these protocols rely on homomorphic encryption to compute MACs of shares. Their distributed homomorphic decryption sub-protocols cannot tolerate dropout in a threshold manner directly and therefore are not suitable for FL settings where dropout is common.

Machine learning based on cryptographic protocols. There are two lines of the cryptographic research regarding privacy and verifiability issues in machine learning, i.e., secure model prediction and secure model training. For secure model prediction, the goal is to securely query a model without revealing model inputs and the information of the model. All works [33], [34], [35], [36], [37] require that the model being queried was well-trained in advance, which differs from the motivation of FL.

For secure model training, there are several works [38], [39], [40] being proposed to train a model while guarantee integrity of the derived model and the privacy of users. Unfortunately, these works will result in expensive overhead when applied to FL settings. For example, Mohassel et al. [38] proposed a framework to convert between any two kinds of secret shares and built up general protocols to train a neural network, where each client is supposed to share each entry of its gradient vector. The communication overhead will blow up as the dimension of vector increases. In addition, as the number of clients increases, to preserve the robustness against dropout, the size of the share held by each client blows up as well.

There are also some works [41], [42] based on homomorphic encryption (HE) which address input privacy only. These works rely on a stronger adversarial model where the 


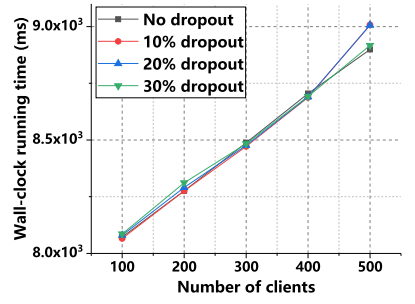

(a)

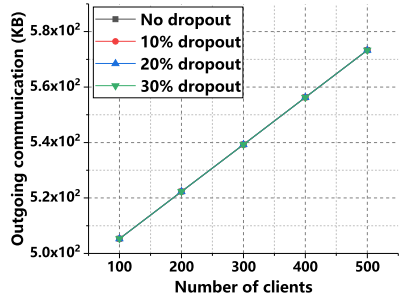

(b)

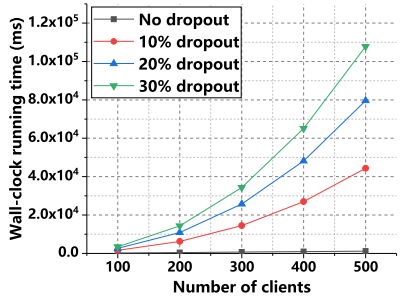

(c)

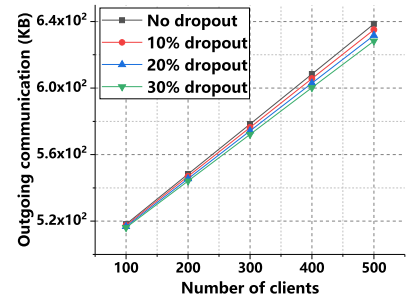

(d)

Fig. 8. Total computation and outgoing communication overhead, as the number of clients increases. (a) Computation overhead per client. (b) Outgoing communication overhead per client. (c) Computation overhead of the server. (d) Outgoing communication overhead of the server (for a single client). The dimension of gradient vectors is set to $100 \mathrm{~K}$ and the batch size is 1 . Assume Case I dropout.

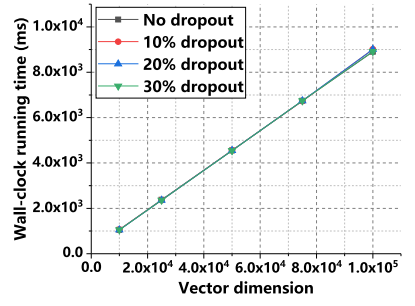

(a)

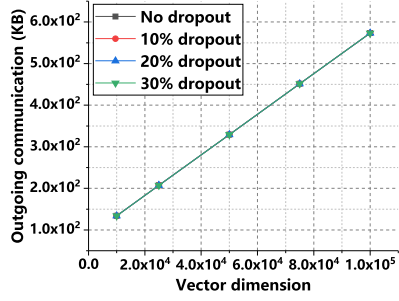

(b)

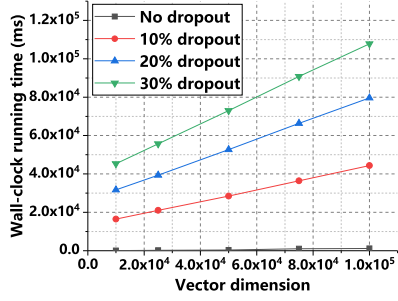

(c)

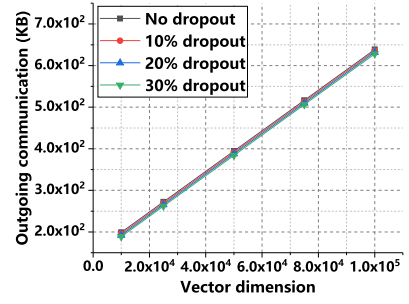

(d)

Fig. 9. Total computation and outgoing communication overhead, as the dimension of gradient vectors increases. (a) Computation overhead per client. (b) Outgoing communication overhead per client. (c) Computation overhead of the server. (d) Outgoing communication overhead of the server (for a single client). The number of clients is set to 500 and the batch size is 1 . Assume Case I dropout.

secret decryption key of encrypted gradients is only known to all clients and these clients are honest. However, in our adversarial model (Section II-C), the adversary can acquire this key from controlled corrupted clients and decrypts all encrypted gradients, which undermines the security of [41], [42]. It is possible to secret share the decryption key (in a threshold manner, e.g., [43], [44]) among all clients to mitigate this issue. Unfortunately, such a construction complicates the decryption of encrypted gradients and, in the FL setting where dropout is common, will significantly increase the size of key shares and the communication between the server and a client as the number of clients increases. So, we believe the protocol based on double-masking is more efficient than that based on HE. In addition, HE is a primitive that can be used to address input privacy only. To achieve both input privacy and verifiability, some other cryptographic primitives (e.g., zero-knowledge proof) are required. This paper finds such a primitive that can be used to replace time-consuming zeroknowledge proof adopted by [8].

To the best of our knowledge, there is only one work [8] for secure verifiable aggregation in FL. However, this work adopts heavy zero-knowledge proof, which results in impractical performance and unaffordable communication overhead.

Differential privacy. A notable fact is that privacypreserving cryptographic protocols cannot fully prevent privacy disclosure in machine learning. It is known that privacy disclosure can be resulted from the statistical characteristics (e.g., confidence information, prediction outcome) of machine learning algorithms. For example, there are some black-box privacy attacks [22], [23], [24], [25], [26] leveraging these statistical characteristics. Treating cryptographic protocols as black-box oracles, such black-box attacks can be applied to the machine learning paradigms even if they are securely realized by cryptographic protocols. That is, as long as these protocols implement the functionality of machine learning, the statistical leakage is inevitable. A possible defense is to combine the cryptographic protocols with differential privacy [45], [46], [47], [48].

Differentially private federated learning [49], [50], [51], [52], [53] has been extensively studied in the literature. A few of existing works [49], [53] consider how to combine differentially private mechanisms with multiparty computation to protect not only the statistical characteristics but also the raw inputs of all honest clients. However, these works do not address the verifiability issue in FL. In this work, we are devoted to fix this issue of FL with practical overhead and differential privacy is not the primary goal. A straightforward way to make our protocol differentially private is to add Gaussian or Laplacian noises to original gradients and then regard the noisy gradients as inputs to our protocol, although it might not achieve the best utility of the global model. An interesting direction for future work would be to explore how to design an optimal differentially private mechanism that is compatible with our practical secure verifiable aggregation protocol.

Byzantine-robust aggregation. A Byzantine client in FL can send arbitrary values to the aggregation server to influence model convergence. To deal with this issue, one popular mitigation is to use Byzantine-robust aggregation, which is mostly related to our aggregation protocol. There are several alternative aggregation mechanisms [54], [55], [56], [57] to the standard federated averaging to ensure that convergence is not significantly influenced by Byzantine clients, in which [57] is for non-IID settings. As noted in [58], it is quite challenging to devise a Byzantine-robust aggregation mechanism for non-IID FL datasets. 
Byzantine-robust aggregation can be regarded as a supplement to the secure verifiable aggregation based on cryptographic protocols since the latter guarantees only input privacy and the integrity of aggregation with respect to the used inputs. In other words, secure verifiable aggregation cannot prevent Byzantine clients to use malformed inputs to do harm to either the training process or the resulting global model.

However, how to combine Byzantine-robust aggregation [54], [55], [56], [57] with secure verifiable aggregation is still an open problem. There are several technical challenges to be addressed by future work. First, these works adopt a completely different adversarial model to that of secure verifiable aggregation (e.g., Section II-C). That is, Byzantine aggregation assume only Byzantine clients that send arbitrary values to an honest aggregation server and does not consider the case where the server is corrupted. Second, these works implicitly assume the aggregation server has access to the plaintext stochastic gradients uploaded by clients, which violates input privacy. Since Byzantine-robust aggregation mechanisms usually adopt more complicated arithmetic (e.g., median or trimmed mean) than federated averaging, it is challenging to devise a tailored dropout-tolerant protocol for these mechanisms to achieve input privacy and verifiability additionally.

\section{CONCLUSION}

In this paper, we studied how to realize verifiable aggregation in FL in a communication-efficient and fast way, and proposed a protocol named VERIFL. We show by experiments that VERIFL is capable of dealing with (i) highdimensional gradient vectors, (ii) a large number of clients, and (iii) high dropout rate in real-world applications with practical performance. Notably, in VERIFL, the verification cost in terms of the outgoing communication is independent of the gradient dimension, resulting in $110.6 \times$ improvement in communication even with $5 \times$ larger gradient vectors in our experiments.

\section{REFERENCES}

[1] H. B. McMahan, E. Moore, D. Ramage, S. Hampson et al., "Communication-efficient learning of deep networks from decentralized data," arXiv preprint arXiv:1602.05629, 2016.

[2] J. Konečnỳ, H. B. McMahan, F. X. Yu, P. Richtárik, A. T. Suresh, and D. Bacon, "Federated learning: Strategies for improving communication efficiency," arXiv preprint arXiv:1610.05492, 2016.

[3] K. Bonawitz, H. Eichner, W. Grieskamp, D. Huba, A. Ingerman, V. Ivanov, C. Kiddon, J. Konecny, S. Mazzocchi, H. B. McMahan et al., "Towards federated learning at scale: System design," arXiv preprint arXiv:1902.01046, 2019.

[4] B. Hitaj, G. Ateniese, and F. Perez-Cruz, "Deep models under the gan: information leakage from collaborative deep learning," in Proceedings of the 2017 ACM SIGSAC Conference on Computer and Communications Security. ACM, 2017, pp. 603-618.

[5] L. Melis, C. Song, E. De Cristofaro, and V. Shmatikov, "Exploiting unintended feature leakage in collaborative learning," in 2019 IEEE Symposium on Security and Privacy (SP). IEEE, 2019, pp. 691-706.

[6] M. Nasr, R. Shokri, and A. Houmansadr, "Comprehensive privacy analysis of deep learning: Passive and active white-box inference attacks against centralized and federated learning," in 2019 IEEE Symposium on Security and Privacy (SP). IEEE, 2019, pp. 739-753.

[7] K. Bonawitz, V. Ivanov, B. Kreuter, A. Marcedone, H. B. McMahan, S. Patel, D. Ramage, A. Segal, and K. Seth, "Practical secure aggregation for privacy-preserving machine learning," in Proceedings of the 2017 ACM SIGSAC Conference on Computer and Communications Security. ACM, 2017, pp. 1175-1191.
[8] G. Xu, H. Li, S. Liu, K. Yang, and X. Lin, "Verifynet: Secure and verifiable federated learning," IEEE Transactions on Information Forensics and Security, vol. 15, pp. 911-926, 2019.

[9] E. Bagdasaryan, A. Veit, Y. Hua, D. Estrin, and V. Shmatikov, "How to backdoor federated learning," arXiv preprint arXiv:1807.00459, 2018.

[10] X. Chen, C. Liu, B. Li, K. Lu, and D. Song, "Targeted backdoor attacks on deep learning systems using data poisoning," arXiv preprint arXiv:1712.05526, 2017.

[11] Y. Liu, S. Ma, Y. Aafer, W. Lee, J. Zhai, W. Wang, and X. Zhang, "Trojaning attack on neural networks," in 25th Annual Network and Distributed System Security Symposium, NDSS 2018, San Diego, California, USA, February 18-21, 2018, 2018.

[12] J. Dumford and W. Scheirer, "Backdooring convolutional neural networks via targeted weight perturbations," arXiv preprint arXiv:1812.03128, 2018.

[13] F. Sattler, S. Wiedemann, K.-R. Müller, and W. Samek, "Robust and communication-efficient federated learning from non-iid data," IEEE transactions on neural networks and learning systems, 2019.

[14] I. Damgård, V. Pastro, N. Smart, and S. Zakarias, "Multiparty computation from somewhat homomorphic encryption," in Annual Cryptology Conference. Springer, 2012, pp. 643-662.

[15] M. Keller, V. Pastro, and D. Rotaru, "Overdrive: making spdz great again," in Annual International Conference on the Theory and Applications of Cryptographic Techniques. Springer, 2018, pp. 158-189.

[16] R. Cramer, I. Damgård, D. Escudero, P. Scholl, and C. Xing, "Spd $\mathbb{Z}_{2^{k}}$ : Efficient mpc mod $2^{k}$ for dishonest majority," in Annual International Cryptology Conference. Springer, 2018, pp. 769-798.

[17] I. Damgård, C. Orlandi, and M. Simkin, "Yet another compiler for active security or: efficient mpc over arbitrary rings," in Annual International Cryptology Conference. Springer, 2018, pp. 799-829.

[18] O. Goldreich, S. Micali, and A. Wigderson, "How to play any mental game," in Proceedings of the nineteenth annual ACM symposium on Theory of computing. ACM, 1987, pp. 218-229.

[19] C.-h. Shen et al., "Two-output secure computation with malicious adversaries," in Annual International Conference on the Theory and Applications of Cryptographic Techniques. Springer, 2011, pp. 386405.

[20] B. Kreuter, A. Shelat, and C.-H. Shen, "Billion-gate secure computation with malicious adversaries," in Presented as part of the 21st USENIX Security Symposium (USENIX Security 12), 2012, pp. 285-300.

[21] Y. Huang, J. Katz, and D. Evans, "Efficient secure two-party computation using symmetric cut-and-choose," in Annual Cryptology Conference. Springer, 2013, pp. 18-35.

[22] M. Fredrikson, S. Jha, and T. Ristenpart, "Model inversion attacks that exploit confidence information and basic countermeasures," in Proceedings of the 22nd ACM SIGSAC Conference on Computer and Communications Security, Denver, CO, USA, October 12-16, 2015, 2015, pp. 1322-1333.

[23] Z. Yang, J. Zhang, E. Chang, and Z. Liang, "Neural network inversion in adversarial setting via background knowledge alignment," in Proceedings of the 2019 ACM SIGSAC Conference on Computer and Communications Security, CCS 2019, London, UK, November 11-15, 2019, 2019, pp. 225-240.

[24] R. Shokri, M. Stronati, C. Song, and V. Shmatikov, "Membership inference attacks against machine learning models," in 2017 IEEE Symposium on Security and Privacy, SP 2017, San Jose, CA, USA, May 22-26, 2017, 2017, pp. 3-18.

[25] L. Song, R. Shokri, and P. Mittal, "Membership inference attacks against adversarially robust deep learning models," in 2019 IEEE Security and Privacy Workshops, SP Workshops 2019, San Francisco, CA, USA, May $19-23,2019,2019$, pp. 50-56.

[26] T. Orekondy, B. Schiele, and M. Fritz, "Knockoff nets: Stealing functionality of black-box models," in IEEE Conference on Computer Vision and Pattern Recognition, CVPR 2019, Long Beach, CA, USA, June 1620, 2019, 2019, pp. 4954-4963.

[27] M. Bellare, O. Goldreich, and S. Goldwasser, "Incremental cryptography: The case of hashing and signing," in Annual International Cryptology Conference. Springer, 1994, pp. 216-233.

[28] M. N. Krohn, M. J. Freedman, and D. Mazieres, "On-the-fly verification of rateless erasure codes for efficient content distribution," in IEEE Symposium on Security and Privacy, 2004. Proceedings. 2004. IEEE, 2004, pp. 226-240.

[29] Y. Lindell, How to Simulate It - A Tutorial on the Simulation Proof Technique. Cham: Springer International Publishing, 2017, pp. 277346. 
[30] J. Camenisch, M. Drijvers, T. Gagliardoni, A. Lehmann, and G. Neven, "The wonderful world of global random oracles," in Annual International Conference on the Theory and Applications of Cryptographic Techniques. Springer, 2018, pp. 280-312.

[31] Y. Huang, D. Evans, J. Katz, and L. Malka, "Faster secure two-party computation using garbled circuits." in USENIX Security Symposium, vol. 201, no. 1, 2011, pp. 331-335.

[32] Y. Lindell and B. Pinkas, "An efficient protocol for secure two-party computation in the presence of malicious adversaries," in Annual International Conference on the Theory and Applications of Cryptographic Techniques. Springer, 2007, pp. 52-78.

[33] C. Juvekar, V. Vaikuntanathan, and A. Chandrakasan, "Gazelle: A low latency framework for secure neural network inference," in 27th USENIX Security Symposium (USENIX Security 18), 2018, pp. 1651-1669.

[34] M. S. Riazi, M. Samragh, H. Chen, K. Laine, K. E. Lauter, and F. Koushanfar, "Xonn: Xnor-based oblivious deep neural network inference," in 28th USENIX Security Symposium, USENIX Security 2019, Santa Clara, CA, USA, August 14-16, 2019, 2019, pp. 1501-1518.

[35] J. Liu, M. Juuti, Y. Lu, and N. Asokan, "Oblivious neural network predictions via minionn transformations," in Proceedings of the 2017 ACM SIGSAC Conference on Computer and Communications Security. ACM, 2017, pp. 619-631.

[36] H. Chen, W. Dai, M. Kim, and Y. Song, "Efficient multi-key homomorphic encryption with packed ciphertexts with application to oblivious neural network inference," in Proceedings of the 2019 ACM SIGSAC Conference on Computer and Communications Security. ACM, 2019, pp. 395-412.

[37] R. Gilad-Bachrach, N. Dowlin, K. Laine, K. E. Lauter, M. Naehrig, and J. Wernsing, "Cryptonets: Applying neural networks to encrypted data with high throughput and accuracy," in Proceedings of the 33nd International Conference on Machine Learning, ICML 2016, New York City, NY, USA, June 19-24, 2016, 2016, pp. 201-210.

[38] P. Mohassel and P. Rindal, "Aby 3: a mixed protocol framework for machine learning," in Proceedings of the 2018 ACM SIGSAC Conference on Computer and Communications Security. ACM, 2018, pp. 35-52.

[39] N. Agrawal, A. Shahin Shamsabadi, M. J. Kusner, and A. Gascón, "Quotient: Two-party secure neural network training and prediction," in Proceedings of the 2019 ACM SIGSAC Conference on Computer and Communications Security. ACM, 2019, pp. 1231-1247.

[40] I. Damgård, D. Escudero, T. Frederiksen, M. Keller, P. Scholl, and N. Volgushev, "New primitives for actively-secure mpc over rings with applications to private machine learning," in 2019 IEEE Symposium on Security and Privacy (SP), May 2019, pp. 1102-1120.

[41] L. T. Phong, Y. Aono, T. Hayashi, L. Wang, and S. Moriai, "Privacypreserving deep learning via additively homomorphic encryption," IEEE Trans. Inf. Forensics Secur., vol. 13, no. 5, pp. 1333-1345, 2018.

[42] X. Sun, P. Zhang, J. K. Liu, J. Yu, and W. Xie, "Private machine learning classification based on fully homomorphic encryption," IEEE Trans. Emerg. Top. Comput., vol. 8, no. 2, pp. 352-364, 2020.

[43] D. Boneh, R. Gennaro, S. Goldfeder, A. Jain, S. Kim, P. M. R. Rasmussen, and A. Sahai, "Threshold cryptosystems from threshold fully homomorphic encryption," in Advances in Cryptology - CRYPTO 2018 - 38th Annual International Cryptology Conference, Santa Barbara, CA, USA, August 19-23, 2018, Proceedings, Part I, 2018, pp. 565-596.

[44] A. Jain, P. M. R. Rasmussen, and A. Sahai, "Threshold fully homomorphic encryption," IACR Cryptol. ePrint Arch., vol. 2017, p. 257, 2017.

[45] C. Dwork and A. Roth, "The algorithmic foundations of differential privacy," Found. Trends Theor. Comput. Sci., vol. 9, no. 3-4, pp. 211407,2014

[46] C. Dwork, "Differential privacy: A survey of results," in Theory and Applications of Models of Computation, 5th International Conference, TAMC 2008, Xi'an, China, April 25-29, 2008. Proceedings, 2008, pp. $1-19$.

[47] M. Abadi, A. Chu, I. J. Goodfellow, H. B. McMahan, I. Mironov, K. Talwar, and L. Zhang, "Deep learning with differential privacy," in Proceedings of the 2016 ACM SIGSAC Conference on Computer and Communications Security, Vienna, Austria, October 24-28, 2016, 2016, pp. 308-318.

[48] F. McSherry and K. Talwar, "Mechanism design via differential privacy," in 48th Annual IEEE Symposium on Foundations of Computer Science (FOCS 2007), October 20-23, 2007, Providence, RI, USA, Proceedings, 2007, pp. 94-103.

[49] S. Truex, N. Baracaldo, A. Anwar, T. Steinke, H. Ludwig, R. Zhang, and Y. Zhou, "A hybrid approach to privacy-preserving federated learning," in Proceedings of the 12th ACM Workshop on Artificial Intelligence and Security, 2019, pp. 1-11.
[50] K. Wei, J. Li, M. Ding, C. Ma, H. H. Yang, F. Farokhi, S. Jin, T. Q. Quek, and H. V. Poor, "Federated learning with differential privacy: Algorithms and performance analysis," IEEE Transactions on Information Forensics and Security, 2020.

[51] H. B. McMahan, D. Ramage, K. Talwar, and L. Zhang, "Learning differentially private recurrent language models," in 6th International Conference on Learning Representations, ICLR 2018, Vancouver, BC Canada, April 30 - May 3, 2018, Conference Track Proceedings, 2018.

[52] R. C. Geyer, T. Klein, and M. Nabi, "Differentially private federated learning: A client level perspective," arXiv preprint arXiv:1712.07557, 2017.

[53] T. Ryffel, A. Trask, M. Dahl, B. Wagner, J. Mancuso, D. Rueckert, and J. Passerat-Palmbach, "A generic framework for privacy preserving deep learning," arXiv preprint arXiv:1811.04017, 2018.

[54] Y. Chen, L. Su, and J. Xu, "Distributed statistical machine learning in adversarial settings: Byzantine gradient descent," Proc. ACM Meas. Anal. Comput. Syst., vol. 1, no. 2, pp. 44:1-44:25, 2017.

[55] D. Yin, Y. Chen, K. Ramchandran, and P. L. Bartlett, "Byzantine-robust distributed learning: Towards optimal statistical rates," in Proceedings of the 35th International Conference on Machine Learning, ICML 2018, Stockholmsmässan, Stockholm, Sweden, July 10-15, 2018, 2018, pp. 5636-5645.

[56] P. Blanchard, E. M. E. Mhamdi, R. Guerraoui, and J. Stainer, "Machine learning with adversaries: Byzantine tolerant gradient descent," in Advances in Neural Information Processing Systems 30: Annual Conference on Neural Information Processing Systems 2017, 4-9 December 2017, Long Beach, CA, USA, 2017, pp. 119-129.

[57] L. He, S. P. Karimireddy, and M. Jaggi, "Byzantine-robust learning on heterogeneous datasets via resampling," CoRR, vol. abs/2006.09365, 2020.

[58] P. Kairouz, H. B. McMahan, B. Avent, A. Bellet, M. Bennis, A. N. Bhagoji, K. Bonawitz, Z. Charles, G. Cormode, R. Cummings et al., "Advances and open problems in federated learning," arXiv preprint arXiv:1912.04977, 2019.

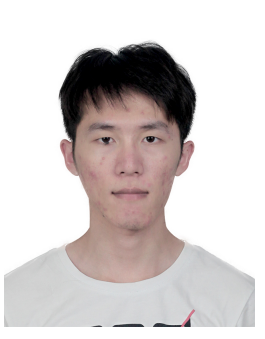

Xiaojie Guo received B.Eng. degree in information security and law in Nankai University in 2018. He is currenting pursuing the master degree in College of Cyber Science, Nankai University, China. His research interests include cryptography, multiparty computation and privacy-preserving machine learning.

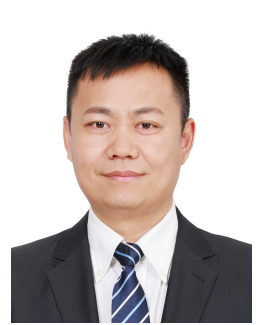

Zheli Liu received the BSc and MSc degrees in computer science from Jilin University, China, in 2002 and 2005, respectively. He received the $\mathrm{PhD}$ degree in computer application from Jilin University in 2009. After a postdoctoral fellowship in Nankai University, he joined the College of Computer and Control Engineering of Nankai University in 2011. Currently, he works at Nankai University as an Associate Professor. His current research interests include applied cryptography and data privacy protection. 


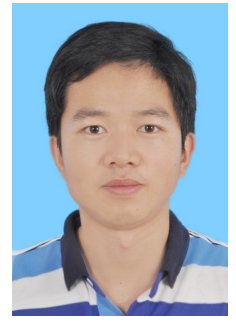

Jin $\mathbf{L i}$ received the BS degree inmathematics from Southwest, University, in 2002 and the $\mathrm{PhD}$ degree in information security from Sun Yat-sen University, in 2007. Currently, he works at Guangzhou University as a Professor. He has been selected as one of science and technology new star in Guangdong province. His research interests include applied cryptography and security in cloud computing. He has published over 50 research papers in refereed international conferences and journals and has served as the program chair or program committee member in many international conferences.

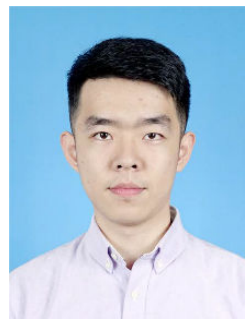

Jiqiang Gao is a master at Nankai University in China. In 2018, he received the B.S degree in information security from Nankai University. His main research interests include protocol security about machine learning algorithm, distributed machine learning, adversarial machine learning. The interests mainly focus on the attack and defense on different machine learning algorithms.

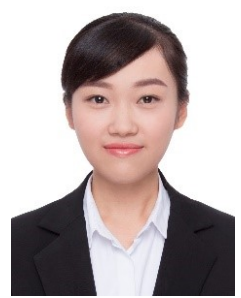

Boyu Hou is a postgraduate from Nankai University, Computer Science Department, Database and Information System Lab, and majors in Computer Science. Her main research field is AI security, include secure distributed machine learning algorithms, adversarial machine learning, attack and defense method on machine learning algorithms.

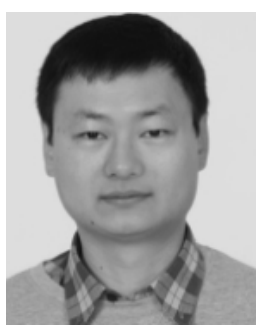

Changyu Dong received the Ph.D. degree from Imperial College London. He is currently a Senior Lecturer with the School of Computing, Newcastle University. He has authored over 30 publications in international journals and conferences. His research interests include applied cryptography, trust management, data privacy, and security policies. His recent work focuses mostly on designing practical secure computation protocols. The application domains include secure cloud computing and privacy preserving data mining.

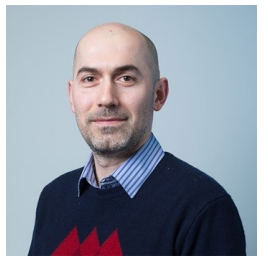

Thar Baker is a Senior Lecturer in Software Systems in the Department of Computer Science at the Faculty of Engineering and Technology. He has received his $\mathrm{PhD}$ in Autonomic Cloud Applications from LJMU in 2010. Dr Baker has published numerous refereed research papers in multidisciplinary research areas including: Cloud Computing, Distributed Software Systems, Big Data, Algorithm Design, Green and Sustainable Computing, and Autonomic Web Science. He has been actively involved as member of editorial board and review committee for a number peer reviewed international journals, and is on programme committee for a number of international conferences. Dr. Baker was appointed as Expert Evaluater in the European FP7 Connected Communities CONFINE project (2012-2015). He worked as Lecturer in the Department of Computer Science at Manchester Metropolitan University (MMU) in 2011. 